\title{
Finite BMS transformations
}

\author{
Glenn Barnich ${ }^{a}$ and Cédric Troessaert ${ }^{b}$ \\ a Physique Théorique et Mathématique, \\ Université Libre de Bruxelles and International Solvay Institutes, \\ Campus Plaine C.P. 231, B-1050 Bruxelles, Belgium \\ ${ }^{b}$ Centro de Estudios Cientificos (CECs), \\ Arturo Prat 514, Valdivia, Chile \\ E-mail: gbarnich@ulb.ac.be, troessaert@cecs.cl
}

ABSTRACT: The action of finite BMS and Weyl transformations on the gravitational data at null infinity is worked out in three and four dimensions in the case of an arbitrary conformal factor for the boundary metric induced on Scri.

Keywords: Classical Theories of Gravity, Gauge Symmetry, Gauge-gravity correspondence

ARXiv EPRINT: 1601.04090 


\section{Contents}

1 Introduction 1

2 Adapted Cartan formulation 3

3 Newman-Penrose formalism in $\mathbf{3 d} \quad 3$

4 3d asymptotically AdS spacetimes at spatial infinity 5

4.1 Fefferman-Graham solution space 5

4.2 Residual gauge symmetries 8

4.3 Action of conformal and Weyl group 10

$5 \quad 3 d$ asymptotically flat spacetimes at null infinity $\quad 11$

$\begin{array}{lll}5.1 & \text { Solution space } & 11\end{array}$

$\begin{array}{lll}5.2 & \text { Residual gauge symmetries } & 12\end{array}$

$\begin{array}{lll}5.3 & \text { Combined BMS3 and Weyl group } & 15\end{array}$

$\begin{array}{ll}5.4 \text { Action on solution space } & 16\end{array}$

$6 \quad 4 d$ asymptotically flat spacetimes at null infinity $\quad 17$

$\begin{array}{lll}6.1 & \text { Newman-Penrose formalism in 4d } & 17\end{array}$

6.2 Newman-Unti solution space 20

6.3 Residual gauge symmetries 24

6.4 Combined extended BMS4 group with complex rescalings 29

6.5 Action on solution space 31

7 Discussion $\quad 35$

$\begin{array}{ll}\text { A Newman-Penrose field equations in 3d } & 36\end{array}$

B Additional transformation laws in 4d 36

\section{Introduction}

There are two main applications of two dimensional conformal invariance [1]. The first consists in using Ward identities associated to infinitesimal symmetry transformations in order to constrain correlation functions. In the second application, starting from known quantities in a given domain, the finite transformations are used to generate the corresponding quantities pertaining to the transformed domain (see e.g. [2]). In this case, the Schwarzian derivative occuring in the transformation law of the energy-momentum tensor plays a crucial role. 
For four-dimensional asymptotically flat spacetimes at null infinity, an extension of the globally well-defined symmetry group [3-5] in terms of locally defined infinitesimal transformations has been proposed and studied in [6-10]. In particular, their relevance for gravitational scattering has been conjectured. Physical implications in terms of Ward identities for soft gravitons have subsequently been developed in [11-14].

The aim of the present paper is to derive the finite transformations necessary for the second application. In particular for instance, if one knows the theory in the form of an asymptotic solution to classical general relativity for the standard topology $S^{2} \times \mathbb{R}$ of $\mathscr{I}^{+}$, one can use the transformation laws to get the solution on a cylinder times a line. Particular aspects of such mappings in general relativity have been discussed previously for instance in [15-17]. More concretely, in the present paper we will work out the transformation laws of asymptotic solution space and the analog of the Schwarzian derivative for finite extended $\mathrm{BMS}_{4}$ transformations and local time-dependent complex Weyl rescalings. Whereas the former corresponds to the residual symmetry group, the latter represents the natural ambiguity in the definition of asymptotically flat spacetimes in terms of conformal compactifications $[18,19]$.

As a warm-up, we start by re-deriving the known finite transformations in three dimensions in the asymptotically anti-de Sitter and flat cases. In the former case, one recovers the Schwarzian derivative as an application of the $\mathrm{AdS}_{3} / \mathrm{CFT}_{2}$ correspondence [20, 21]. In the latter case, one obtains the finite transformation laws for the Bondi mass and angular momentum aspects that have been previously obtained by directly integrating the infinitesimal transformations [22]. In both these three dimensional cases, these results are generalized to include local Weyl transformations. In other words, we are working out the action of finite Penrose-Brown-Henneaux transformations in the terminology of [23, 24].

Explicit computations are done in the framework of the Newman-Penrose formalism $[25,26]$, as applied to asymptotically flat four dimensional spacetimes at null infinity in $[27,28]$. Standard reviews are [29-33].

To summarize the results for the simplest case when computations are done with respect to the Riemann sphere, i.e., when the metric on $\mathscr{I}^{+}$is taken as $d \bar{s}^{2}=0 d \widetilde{u}^{2}-2 d \zeta d \bar{\zeta}$, the extended $\mathrm{BMS}_{4}$ group consists of superrotations $\zeta=\zeta\left(\zeta^{\prime}\right), \bar{\zeta}=\bar{\zeta}\left(\bar{\zeta}^{\prime}\right)$ together with supertranslations $\widetilde{u}^{\prime}=\left(\frac{\partial \zeta}{\partial \zeta^{\prime}} \frac{\partial \bar{\zeta}}{\partial \vec{\zeta}^{\prime}}\right)^{-\frac{1}{2}}[\widetilde{u}+\beta(\zeta, \bar{\zeta})]$. In particular, the asymptotic part of the shear, the news, and the Bondi mass aspect transform as

$$
\begin{aligned}
\sigma_{R}^{\prime 0} & =\left(\frac{\partial \zeta}{\partial \zeta^{\prime}}\right)^{-\frac{1}{2}}\left(\frac{\partial \bar{\zeta}}{\partial \bar{\zeta}^{\prime}}\right)^{\frac{3}{2}}\left[\sigma_{R}^{0}+\bar{\partial}^{2} \beta+\frac{1}{2}\left\{\bar{\zeta}^{\prime}, \bar{\zeta}\right\}(\tilde{u}+\beta)\right], \\
\dot{\sigma}_{R}^{\prime 0} & =\left(\frac{\partial \bar{\zeta}}{\partial \bar{\zeta}^{\prime}}\right)^{2}\left[\dot{\sigma}_{R}^{0}+\frac{1}{2}\left\{\bar{\zeta}^{\prime}, \bar{\zeta}\right\}\right], \\
(-4 \pi G) M_{R}^{\prime}= & \left(\frac{\partial \zeta}{\partial \zeta^{\prime}} \frac{\partial \bar{\zeta}}{\partial \bar{\zeta}^{\prime}}\right)^{\frac{3}{2}}\left[(-4 \pi G) M_{R}+\bar{\partial}^{2} \partial^{2} \beta+\frac{1}{2}\left\{\bar{\zeta}^{\prime}, \bar{\zeta}\right\}\left(\bar{\sigma}_{R}^{0}+\partial^{2} \beta\right)\right. \\
& \left.+\frac{1}{2}\left\{\zeta^{\prime}, \zeta\right\}\left(\sigma_{R}^{0}+\bar{\partial}^{2} \beta\right)+\frac{1}{4}\left\{\bar{\zeta}^{\prime}, \bar{\zeta}\right\}\left\{\zeta^{\prime}, \zeta\right\}(\widetilde{u}+\beta)\right],
\end{aligned}
$$

where $\{\cdot, \cdot\}$ denotes the Schwarzian derivative. 


\section{Adapted Cartan formulation}

In the Cartan formulation of general relativity, the fundamental fields are on the one hand, a vielbein, $e_{a}{ }^{\mu}$, together with its inverse $e^{a}{ }_{\mu}$ and associated metric $g_{\mu \nu}=e^{a}{ }_{\mu} \eta_{a b} e^{b}{ }_{\nu}$, where $\eta_{a b}$ is constant and, on the other hand, a Lorentz connection satisfying the metricity condition $\nabla_{a} \eta_{b c}=0, \Gamma_{a b c}=\eta_{a d} \Gamma_{b c}^{d}=\Gamma_{[a b] c}$. Indices are lowered and raised with $\eta_{a b}$ and $g_{\mu \nu}$ and their inverses. The associated connection 1-form is $\Gamma^{a}{ }_{b}=\Gamma_{b c}^{a} e^{c}$ with $e^{c}=e^{c}{ }_{\mu} d x^{\mu}$. The torsion and curvature 2-forms are given by $T^{a}=d e^{a}+\Gamma_{b}^{a} \wedge e^{b}, R_{b}^{a}=d \Gamma_{b}^{a}+\Gamma^{a}{ }_{c} \wedge \Gamma_{b}^{c}$.

Local Lorentz transformations are described by matrices $\Lambda_{a}^{b}(x)$ with $\Lambda^{a}{ }_{c} \Lambda_{b}{ }^{c}=\delta_{b}^{a}$. Under combined frame and coordinate transformations, referred to as gauge transformations below, the basic variables transform as

$$
\begin{aligned}
e_{a}^{\prime \mu}\left(x^{\prime}\right) & =\left(\Lambda_{a}{ }^{b} e_{b}{ }^{\nu} \frac{\partial x^{\prime \mu}}{\partial x^{\nu}}\right)(x), \\
\Gamma_{a b c}^{\prime}\left(x^{\prime}\right) & =\left(\Lambda_{a}{ }^{d}\left[\Lambda_{b}{ }^{e} \Gamma_{d e f}+e_{f}\left(\Lambda_{b d}\right)\right] \Lambda_{c}^{f}\right)(x),
\end{aligned}
$$

where the last expression is equivalent to the transformation law for the connection 1-form, $\Gamma^{\prime a}{ }_{b}=\Lambda_{c}^{a}{ }_{c}^{c}{ }_{d} \Lambda_{b}{ }^{d}+\Lambda_{c}^{a}{ }_{c} d \Lambda_{b}{ }^{c}$ and $e_{a}=e_{a}{ }^{\mu} \frac{\partial}{\partial x^{\mu}}$.

Equations of motion deriving from the variational principle

$$
S[e, \Gamma]=\frac{1}{16 \pi G} \int d^{d} x e\left(R_{a b c d} \eta^{a c} \eta^{b d}-2 \Lambda\right),
$$

are equivalent to $T^{a}=d e^{a}+\Gamma_{b}^{a} e^{b}=0$ and Einstein's equations, $G_{a b}+\Lambda \eta_{a b}=0$. Together with the metricity condition, the former implies

$$
\Gamma_{a b c}=\frac{1}{2}\left(D_{b a c}+D_{c a b}-D_{a b c}\right),
$$

where the structure functions are defined by $D_{a b}^{c} e_{c}=\left(e_{a}\left(e_{b}^{\mu}\right)-e_{b}\left(e_{a}^{\mu}\right)\right) \frac{\partial}{\partial x^{\mu}}$. Conversely, $T^{a}=0$ is equivalent to $D_{c a b}=-2 \Gamma_{c[a b]}$.

\section{Newman-Penrose formalism in 3d}

In three dimensions with $(+,-,-)$ signature, we use

$$
\eta_{a b}=\left(\begin{array}{ccc}
0 & 1 & 0 \\
1 & 0 & 0 \\
0 & 0 & -\frac{1}{2}
\end{array}\right),
$$

and the triad $e_{a}=(l, n, m)$ with associated directional covariant derivatives denoted by $(D, \Delta, \delta)$. Note that this choice of $\eta_{a b}$ implies different conventions and normalizations than used in previous works. In particular,

$$
g^{\mu \nu}=l^{\mu} n^{\nu}+l^{\nu} n^{\mu}-2 m^{\mu} m^{\nu}, \quad \nabla_{a}=n_{a} D+l_{a} \Delta-2 m_{a} \delta .
$$


In this case, the spin connection can be dualized, $\omega_{c \mu}=\frac{1}{4} \Gamma^{a b}{ }_{\mu} \epsilon_{a b c}, \Gamma^{a b}{ }_{\mu}=\epsilon^{a b c} \omega_{c \mu}$ with $\epsilon_{123}=1$ and $\epsilon^{a b c}=\eta^{a d} \eta^{b e} \eta^{c f} \epsilon_{\text {def }}$. The 9 real spin coefficients are defined by

\begin{tabular}{|c|c|c|c|}
\hline$\nabla$ & $m^{a} \nabla l_{a}$ & $n^{a} \nabla l_{a}$ & $-m^{a} \nabla n_{a}$ \\
\hline$D$ & $\kappa=\Gamma_{311}=\omega^{2}{ }_{1}$ & $\epsilon=\Gamma_{211}=-\omega^{3}{ }_{1}$ & $\pi=-\Gamma_{321}=\omega^{1}{ }_{1}$ \\
\hline$\Delta$ & $\tau=\Gamma_{312}=\omega^{2}{ }_{2}$ & $\gamma=\Gamma_{212}=-\omega^{3}{ }_{2}$ & $\nu=-\Gamma_{322}=\omega^{1}{ }_{2}$ \\
\hline$\delta$ & $\sigma=\Gamma_{313}=\omega^{2}{ }_{3}$ & $\beta=\Gamma_{213}=-\omega^{3}{ }_{3}$ & $\mu=-\Gamma_{323}=\omega^{1}{ }_{3}$ \\
\hline
\end{tabular}

(see also e.g. [34] for slightly different conventions). It follows that

$$
\begin{aligned}
& D l=\epsilon l-2 \kappa m, \quad \Delta l=\gamma l-2 \tau m, \quad \delta l=\beta l-2 \sigma m, \\
& D n=-\epsilon n+2 \pi m, \quad \Delta n=-\gamma n+2 \nu m, \quad \delta n=-\beta n+2 \mu m, \\
& D m=\pi l-\kappa n, \quad \Delta m=\nu l-\tau n, \quad \delta m=\mu l-\sigma n \text {. }
\end{aligned}
$$

In order to describe Lorentz transformations, one associates to a real vector $v=v^{a} e_{a}$ a $2 \times 2$ symmetric matrix $\hat{v}=v^{a} \widehat{j}_{a}$, where $\widehat{j}_{a}$ are chosen as

$$
\widehat{j_{1}}=\left(\begin{array}{ll}
1 & 0 \\
0 & 0
\end{array}\right), \widehat{j}_{2}=\left(\begin{array}{ll}
0 & 0 \\
0 & 1
\end{array}\right), \widehat{j}_{3}=\frac{1}{2}\left(\begin{array}{ll}
0 & 1 \\
1 & 0
\end{array}\right),
$$

so that

$$
\operatorname{det} \widehat{v}=\frac{1}{2} \eta_{a b} v^{a} v^{b}, \quad \widehat{j}_{a} \epsilon \widehat{j}_{b}=\frac{1}{2}\left(\eta_{a b} \epsilon-\epsilon_{a b c} \widehat{j}^{c}\right), \quad \widehat{j}^{a} \widehat{j}_{b} \widehat{j}_{a}=\frac{1}{2} \epsilon \widehat{j}_{b} \epsilon
$$

where

$$
\epsilon=\left(\begin{array}{cc}
0 & 1 \\
-1 & 0
\end{array}\right)
$$

For $g \in \mathrm{SL}(2, \mathbb{R})$, one considers the transformation

$$
\widehat{g}_{a} g^{T} v^{a}=\widehat{j}_{a} \Lambda_{b}^{a} v^{b}, \quad g^{T} \epsilon=\epsilon g^{-1} .
$$

More explicitly, if

$$
g=\left(\begin{array}{ll}
a & b \\
c & d
\end{array}\right)
$$

with $a d-b c=1$ and $a, b, c, d \in \mathbb{R}$, then

$$
\Lambda_{b}^{a}=\left(\begin{array}{ccc}
a^{2} & b^{2} & a b \\
c^{2} & d^{2} & c d \\
2 a c & 2 b d & a d+b c
\end{array}\right), \Lambda_{a}^{b}=\left(\begin{array}{ccc}
d^{2} & c^{2} & -2 c d \\
b^{2} & a^{2} & -2 a b \\
-b d-a c & a d+b c
\end{array}\right)
$$

where the first index is the lign index. $\mathrm{SL}(2, \mathbb{R})$ group elements will be parametrized as

$$
g=\left(\begin{array}{cc}
1 & 0 \\
-B & 1
\end{array}\right)\left(\begin{array}{cc}
1 & -A \\
0 & 1
\end{array}\right)\left(\begin{array}{cc}
e^{-E / 2} & 0 \\
0 & e^{E / 2}
\end{array}\right)=\left(\begin{array}{cc}
e^{-E / 2} & -A e^{E / 2} \\
-B e^{-E / 2} & (1+A B) e^{E / 2}
\end{array}\right) .
$$

Using $\omega^{a}=\frac{1}{2} \epsilon^{a b c} \Gamma_{b c}$ and the transformation law of the Lorentz connection given below (2.1), we have

$$
\omega^{a}=\Lambda_{b}^{a} \omega^{b}+\frac{1}{2} \epsilon^{a b c} \Lambda_{b}{ }^{d} d \Lambda_{c d} .
$$


In terms of $\hat{\omega}=\hat{j}_{a} \omega^{a}$, this is equivalent to

$$
\hat{\omega}^{\prime}=g \hat{\omega} g^{T}-g \epsilon d g^{T}
$$

Explicitly, for the spin coefficients encoded in

$$
\hat{\omega}_{1}=\left(\begin{array}{cc}
\pi & -\frac{1}{2} \epsilon \\
-\frac{1}{2} \epsilon & \kappa
\end{array}\right), \quad \hat{\omega}_{2}=\left(\begin{array}{cc}
\nu & -\frac{1}{2} \gamma \\
-\frac{1}{2} \gamma & \tau
\end{array}\right), \quad \hat{\omega}_{3}=\left(\begin{array}{cc}
\mu & -\frac{1}{2} \beta \\
-\frac{1}{2} \beta & \sigma
\end{array}\right),
$$

one finds

$$
\hat{\omega}_{a}^{\prime}=\Lambda_{a}^{c} g \hat{\omega}_{c} g^{T}-g \epsilon e_{a}^{\prime}\left(g^{T}\right) .
$$

In this case, Einstein's equations are equivalent to

$$
d \hat{e}-2 \hat{\omega} \epsilon \hat{e}=0, \quad d \hat{\omega}-\hat{\omega} \epsilon \hat{\omega}-\frac{\Lambda}{2} \hat{e} \epsilon \hat{e}=0 .
$$

Alternatively, one can use $\breve{v}=\hat{v} \epsilon$ in order to describe real vectors by traceless $2 \times 2$ matrices. The associated basis is

$$
\check{j}_{1}=\left(\begin{array}{ll}
0 & 1 \\
0 & 0
\end{array}\right), \breve{j}_{2}=\left(\begin{array}{cc}
0 & 0 \\
-1 & 0
\end{array}\right), \breve{j}_{3}=\frac{1}{2}\left(\begin{array}{cc}
-1 & 0 \\
0 & 1
\end{array}\right),
$$

so that

$$
\operatorname{tr} \breve{v}^{2}=\eta_{a b} v^{a} v^{b}, \quad \breve{j}_{a} \breve{j}_{b}=-\frac{1}{2}\left(\eta_{a b}+\epsilon_{a b c} \breve{j}^{c}\right), \quad \check{j}^{a} \check{j}_{b} \breve{j}_{a}=\frac{1}{2} \breve{j}_{b} .
$$

In this case, we have

$$
g \breve{j}_{a} g^{-1} v^{a}=\breve{j}_{a} \Lambda^{a}{ }_{b} v^{b}, \quad \breve{\omega}^{\prime}=g \breve{\omega}^{-1}-d g g^{-1}, \quad \breve{\omega}_{a}^{\prime}=\Lambda_{a}^{c} g \breve{\omega}_{c} g^{-1}-e_{a}^{\prime}(g) g^{-1},
$$

where

$$
\breve{\omega}_{1}=\left(\begin{array}{cc}
\frac{1}{2} \epsilon & \pi \\
-\kappa & -\frac{1}{2} \epsilon
\end{array}\right), \quad \breve{\omega}_{2}=\left(\begin{array}{cc}
\frac{1}{2} \gamma & \nu \\
-\tau & -\frac{1}{2} \gamma
\end{array}\right), \quad \breve{\omega}_{3}=\left(\begin{array}{cc}
\frac{1}{2} \beta & \mu \\
-\sigma & -\frac{1}{2} \beta
\end{array}\right),
$$

and

$$
d \check{e}-2 \breve{\omega} \breve{e}=0, \quad d \breve{\omega}-\breve{\omega} \breve{\omega}-\frac{\Lambda}{2} \widetilde{e e}=0 .
$$

\section{3d asymptotically AdS spacetimes at spatial infinity}

\subsection{Fefferman-Graham solution space}

In the $\mathrm{AdS}_{3}$ case, $\Lambda=-L^{-2} \neq 0$, we start by rederiving the general solution to the equations of motion in the context of the Newman-Penrose formalism. We will recover the on-shell bulk metric of [35], but with an arbitrary conformal factor for the boundary metric [21] (see also section 2 of [7] in the current context).

The analog of the Fefferman-Graham gauge fixing is to assume that

$$
\mu=\beta=\sigma=0 .
$$

which is equivalent to $\Gamma_{a b 3}=0$ and can be achieved by a local Lorentz transformation. This means that the triad is parallely transported along $m$ and that $m$ is the generator of 
an affinely parametrized spatial geodesic. In this case, $\nabla_{[a} m_{b]}=n_{[a} l_{b]}(\pi+\tau)$ so that $m$ is hypersurface orthonormal if and only it is a gradient, which in turn is equivalent to

$$
\pi=-\tau .
$$

This condition will also be imposed in the following.

Introducing coordinates $x^{\mu}=\left(x^{+}, x^{-}, \rho\right), \mu=1,2,3$ such that $m$ is normal to the surfaces $\rho=$ cte and the coordinate $\rho$ is the suitably normalized affine parameter on the geodesic generated by $m$, the triad takes the form

$$
m=\frac{\partial}{\partial \rho}, \quad l=l^{a} \frac{\partial}{\partial x^{a}}, \quad n=n^{a} \frac{\partial}{\partial x^{a}} .
$$

where $a=(+,-)$. The associated cotriad is

$$
e^{1}=\frac{\epsilon_{a b} n^{b} d x^{a}}{e}, \quad e^{2}=\frac{\epsilon_{a b} l^{a} d x^{b}}{e}, \quad e^{3}=d \rho, \quad e=\epsilon_{a b} l^{a} n^{b},
$$

where $\epsilon_{+-}=1=-\epsilon_{-+}$and $\epsilon_{ \pm \pm}=0$. In order to compare with the general solution given in [7], one introduces an alternative radial coordinate $r=e^{\frac{\rho}{\sqrt{2} L}}$, in terms of which

$$
m=\frac{r}{\sqrt{2} L} \frac{\partial}{\partial r}, \quad e^{3}=\sqrt{2} \frac{L}{r} d r .
$$

Under these assumptions, the Newman-Penrose field equations (A.1)-(A.12) can be solved exactly. Indeed, the three equations (A.1), (A.7) and (A.9) reduce to the system

$$
\delta \kappa=2 \tau \kappa, \quad \delta \nu=2 \tau \nu, \quad \delta \tau=\tau^{2}-\kappa \nu-\frac{1}{2 L^{2}},
$$

which is solved by introducing the complex combinations $\mathcal{L}_{ \pm}=\tau \pm i \sqrt{\nu \kappa}$. The general solution is given by

$$
\begin{aligned}
& \tau=-\pi=\frac{-1}{\sqrt{2} L k}\left(r^{4}-C_{1}^{2}+C_{2} C_{3}\right), \quad \kappa=\frac{-\sqrt{2} C_{2} r^{2}}{L k}, \quad \nu=\frac{\sqrt{2} C_{3} r^{2}}{L k}, \\
& k=r^{4}-2 C_{1} r^{2}+C_{1}^{2}-C_{2} C_{3} .
\end{aligned}
$$

The last two radial equations involving the spin coefficients, equations (A.3) and (A.8), simplify to

$$
\delta \epsilon=\tau \epsilon+\kappa \gamma, \quad \delta \gamma=\tau \gamma-\nu \epsilon,
$$

and are solved through

$$
\epsilon=C_{4} \frac{r^{3}-C_{1} r}{k}+C_{5} \frac{C_{2} r}{k}, \quad \gamma=C_{5} \frac{r^{3}-C_{1} r}{k}+C_{4} \frac{C_{3} r}{k} .
$$

The last radial equations are (A.11) and (A.12). Their $r$-component are trivially satisfied while their components along $x^{ \pm}$are of the same form than (4.8),

$$
\delta l^{ \pm}=\tau l^{ \pm}+\kappa n^{ \pm}, \quad \delta n^{ \pm}=\tau n^{ \pm}-\nu l^{ \pm},
$$


which leads to

$$
l^{ \pm}=K_{1}^{ \pm} \frac{r^{3}-C_{1} r}{k}+K_{2}^{ \pm} \frac{C_{2} r}{k}, \quad n^{ \pm}=K_{2}^{ \pm} \frac{r^{3}-C_{1} r}{k}+K_{1}^{ \pm} \frac{C_{3} r}{k} .
$$

In these equations, $C_{i}, K_{1}^{ \pm}, K_{2}^{ \pm}$are functions of $x^{a}=x^{ \pm}$alone.

Note that asymptotic invertibility of the triad is controlled by the invertibility of the matrix formed by these functions,

$$
\left(\begin{array}{cc}
K_{1}^{+} & K_{2}^{+} \\
K_{1}^{-} & K_{2}^{-}
\end{array}\right), \quad \epsilon_{a b} K_{1}^{a} K_{2}^{b} \neq 0 .
$$

Using the radial form of the various quantities, equations (A.2) and (A.6) are equivalent to

$$
\begin{aligned}
& K_{1}^{a} \partial_{a} C_{1}-K_{2}^{a} \partial_{a} C_{2}+2 C_{2} C_{5}=0, \\
& K_{1}^{a} \partial_{a} C_{3}-K_{2}^{a} \partial_{a} C_{1}+2 C_{3} C_{4}=0,
\end{aligned}
$$

which then implies that equation (A.4) reduces to

$$
K_{1}^{a} \partial_{a} C_{5}-K_{2}^{a} \partial_{a} C_{4}+2 C_{4} C_{5}+\frac{4}{L^{2}} C_{1}=0,
$$

while the components along $x^{ \pm}$of equation (A.10) become

$$
K_{1}^{a} \partial_{a} K_{2}^{ \pm}-K_{2}^{a} \partial_{a} K_{1}^{ \pm}+C_{5} K_{1}^{ \pm}+C_{4} K_{2}^{ \pm}=0 .
$$

Because of invertibility of the matrix (4.12), equations (4.15) and (4.14) can be used to express $C_{4}, C_{5}$ and $C_{1}$ in terms of $K_{1}^{a}$ and $K_{2}^{a}$. The two equations in (4.13) then become dynamical equations for $C_{2}$ and $C_{3}$. Since we now have treated all Newman-Penrose equations, the solution space is parametrized by $K_{1}^{a}, K_{2}^{a}$ and by initial conditions for $C_{2}$ and $C_{3}$.

In the limit $r$ going to infinity, the triad elements $l$ and $n$ given in (4.11) take the form $l^{ \pm}=r^{-1} K_{1}^{ \pm}+O\left(r^{-3}\right), n^{ \pm}=r^{-1} K_{2}^{ \pm}+O\left(r^{-3}\right)$. With a change of coordinates on the cylinder, we can make the associated asymptotic metric explicitly conformally flat. This amounts to the choice

$$
K_{1}^{+}=0, \quad K_{1}^{-}=\sqrt{2} e^{-\varphi}, \quad K_{2}^{+}=\sqrt{2} e^{-\varphi}, \quad K_{2}^{-}=0 .
$$

Introducing this into equations (4.14) and (4.15), we get

$$
C_{4}=\sqrt{2} e^{-\varphi} \partial_{-} \varphi, \quad C_{5}=-\sqrt{2} e^{-\varphi} \partial_{+} \varphi, \quad C_{1}=L^{2} e^{-2 \varphi} \partial_{-} \partial_{+} \varphi,
$$

while the dynamical equations (4.13) reduce to

$$
\begin{aligned}
& \partial_{+} C_{2}+2 \partial_{+} \varphi C_{2}=L^{2} \partial_{-}\left(e^{-2 \varphi} \partial_{-} \partial_{+} \varphi\right), \\
& \partial_{-} C_{3}+2 \partial_{-} \varphi C_{3}=L^{2} \partial_{+}\left(e^{-2 \varphi} \partial_{-} \partial_{+} \varphi\right) .
\end{aligned}
$$

With the extra conditions (4.16), the space of solutions is parametrised by three functions $\varphi, C_{2}$ and $C_{3}$ defined on the cylinder with coordinates $x^{ \pm}$such that equations (4.18) are valid. These two equations can be integrated directly but we will derive the explicit form of $C_{2}$ and $C_{3}$ in a different way using the action of the asymptotic symmetry group below. 


\subsection{Residual gauge symmetries}

The residual gauge transformations are the finite gauge transformations that preserve the set of asymptotic solutions. Since these transformations map solutions to solutions, once the conditions that determine the asymptotic solution space are preserved, no further restrictions can arise. A gauge transformation is a combination of a local Lorentz transformation and a change of coordinates of the form

$$
r=r\left(r^{\prime}, x^{ \pm}\right), \quad x^{ \pm}=x^{ \pm}\left(r^{\prime}, x^{\prime \pm}\right) .
$$

The unknowns are $A, B, E, r$ and $x^{ \pm}$as functions of $r^{\prime}, x^{\prime \pm}$.

Using the $a=3$ component of the transformation law of the triad,

$$
e_{a}^{\prime \mu} \frac{\partial x^{\nu}}{\partial x^{\prime \mu}}=\Lambda_{a}^{b} e_{b}^{\nu}
$$

the requirement $m^{\prime \mu}=\frac{r^{\prime}}{\sqrt{2} L} \delta_{r^{\prime}}^{\mu}$ is equivalent to

$$
\frac{r^{\prime}}{\sqrt{2} L} \partial_{r^{\prime}} x^{\mu}=-b d l^{\mu}-a c n^{\mu}+(a d+b c) m^{\mu} .
$$

Expanding for each coordinate, we get

$$
\begin{aligned}
\frac{r^{\prime}}{\sqrt{2} L} \frac{\partial x^{+}}{\partial r^{\prime}} & =\frac{\sqrt{2} r}{k} e^{-\varphi}\left(A(1+A B) e^{E} C_{2}+B e^{-E}\left(r^{2}-C_{1}\right)\right), \\
\frac{r^{\prime}}{\sqrt{2} L} \frac{\partial x^{-}}{\partial r^{\prime}} & =\frac{\sqrt{2} r}{k} e^{-\varphi}\left(A(1+A B) e^{E}\left(r^{2}-C_{1}\right)+B e^{-E} C_{3}\right), \\
\frac{r^{\prime}}{\sqrt{2} L} \frac{\partial r}{\partial r^{\prime}} & =(1+2 A B) \frac{r}{\sqrt{2} L} .
\end{aligned}
$$

In order to implement the gauge fixing condition on the new spin coefficients $\breve{\omega}_{3}^{\prime}=0$, we first rewrite the last equation of (3.19) as

$$
g^{-1} e_{a}^{\prime}(g)=\Lambda_{a}^{b} \breve{\omega}_{b}-g^{-1} \breve{\omega}_{a}^{\prime} g .
$$

When $a=3$ this becomes

$$
g^{-1} \delta^{\prime} g=\Lambda_{3}^{b} \breve{\omega}_{b},
$$

and is equivalent to three conditions on the Lorentz parameters,

$$
d \delta^{\prime} a-b \delta^{\prime} c=\Lambda_{3}{ }^{b}\left(\breve{\omega}_{b}\right)_{11}, d \delta^{\prime} b-b \delta^{\prime} d=\Lambda_{3}{ }^{b}\left(\breve{\omega}_{b}\right)_{12}, a \delta^{\prime} c-c \delta^{\prime} a=\Lambda_{3}{ }^{b}\left(\breve{\omega}_{b}\right)_{21} .
$$

When suitably combining these equations, one finds

$$
\begin{aligned}
& \frac{r^{\prime}}{\sqrt{2} L} \frac{\partial B}{\partial r^{\prime}}=A(1+A B) e^{2 E} \kappa+B \tau, \\
& \frac{r^{\prime}}{\sqrt{2} L} \frac{\partial A}{\partial r^{\prime}}=-A(1+A B) \pi-B e^{-2 E} \nu+A^{3}(1+A B) e^{2 E} \kappa+A^{2} B \tau, \\
& \frac{r^{\prime}}{\sqrt{2} L} \frac{\partial E}{\partial r^{\prime}}=A(1+A B) e^{E} \epsilon-B e^{-E} \gamma-2 A^{2}(1+A B) e^{2 E} \kappa-2 A B \tau .
\end{aligned}
$$


The set of equations (4.22) and (4.26) forms a system of differential equations for the radial dependence of the unknown functions. In order to solve it asymptotically, we will assume that the functions have the following asymptotic behavior,

$$
r=O\left(r^{\prime}\right), \quad x^{ \pm}, E=O(1), \quad A, B=O\left(r^{-1}\right) .
$$

Inserting this into the equations, we easily get

$$
\begin{aligned}
B & =B_{0}\left(x^{\prime \pm}\right) r^{\prime-1}+O\left(r^{\prime-3}\right), \quad A=A_{0}\left(x^{ \pm}\right) r^{\prime-1}+O\left(r^{\prime-3}\right), \\
E & =E_{0}\left(x^{\prime \pm}\right)-\left(L e^{-\varphi} \partial_{-} \varphi A_{0}+L e^{-\varphi} \partial_{+} \varphi B_{0}+A_{0} B_{0}\right) r^{\prime-2}+O\left(r^{\prime-4}\right), \\
r & =e^{r_{0}\left(x^{ \pm}\right)} r^{\prime}-A_{0} B_{0} e^{r_{0}} r^{\prime-1}+O\left(r^{\prime-3}\right), \\
x^{+} & =x_{0}^{+}\left(x^{\prime \pm}\right)-L B_{0} e^{-\varphi-E_{0}-r_{0}} r^{\prime-2}+O\left(r^{\prime-4}\right), \\
x^{-} & =x_{0}^{-}\left(x^{\prime \pm}\right)-L A_{0} e^{-\varphi+E_{0}-r_{0}} r^{\prime-2}+O\left(r^{\prime-4}\right),
\end{aligned}
$$

where we have assumed $\frac{r}{r^{\prime}}>0$ asymptotically. At this stage, we have fixed the radial dependence of all the unknown functions and we are left with six functions $A_{0}, B_{0}, E_{0}$, $r_{0}, x_{0}^{ \pm}$of $x^{\prime \pm}$.

We now have to require $m_{\mu}^{\prime}=\frac{\sqrt{2} L}{r^{\prime}} \delta_{\mu}^{r^{\prime}}$. However, since we have inmposed $m^{\prime}=\frac{r^{\prime}}{\sqrt{2} L} \frac{\partial}{\partial r^{\prime}}$, we already have $m_{r^{\prime}}^{\prime}=\frac{\sqrt{2} L}{r^{\prime}}$. This follows from $m_{r^{\prime}}^{\prime}=\Lambda_{b}^{3} e^{b}{ }_{\nu} \frac{\partial x^{\nu}}{\partial r^{\prime}}$ on the one hand and from $\frac{r^{\prime}}{\sqrt{2} L} \frac{\partial x^{\nu}}{\partial r^{\prime}}=\Lambda_{3}{ }^{c} e_{c}{ }^{\nu}$ on the other. For the remaining components of $m_{\mu}^{\prime}$ it is enough to verify that $m_{ \pm^{\prime}}^{\prime}=o\left(r^{\prime 0}\right)$ since solutions are transformed into solutions under local Lorentz and coordinate transformations. Indeed, $d e^{a}+\Gamma^{\prime a} b^{\prime b}=0$, and for $a=3, d e_{3}^{\prime}+\Gamma_{3 b}^{\prime} e^{\prime b}=0$. Contracting with $e_{3}^{\prime \mu}$ then implies that $\frac{r^{\prime}}{\sqrt{2} L} \partial_{r^{\prime}} m_{\nu^{\prime}}-\frac{r^{\prime}}{\sqrt{2} L} \partial_{\nu^{\prime}} \frac{\sqrt{2} L}{r^{\prime}}+\Gamma_{3 b 3}^{\prime} e^{\prime b} \nu^{\prime}-\Gamma_{33 \nu^{\prime}}^{\prime}=0$, which reduces to $\partial_{r^{\prime}} m_{\nu}^{\prime}=-\frac{\sqrt{2} L}{r^{\prime 2}} \delta_{\nu}^{r^{\prime}}$. Extracting the leading order from

$$
e_{\mu}^{\prime 3}=2 a c e_{\nu}^{1} \frac{\partial x^{\nu}}{\partial x^{\prime \pm}}+2 b d e_{\nu}^{2} \frac{\partial x^{\nu}}{\partial x^{\prime \pm}}+(a d+b c) e_{\nu}^{3} \frac{\partial x^{\nu}}{\partial x^{\prime \pm}}
$$

we then get

$$
B_{0} e^{-E_{0}+r_{0}+\varphi} \frac{\partial x_{0}^{-}}{\partial x^{\prime \pm}}+A_{0} e^{E_{0}+r_{0}+\varphi} \frac{\partial x_{0}^{+}}{\partial x^{\prime \pm}}=L \frac{\partial r_{0}}{\partial x^{\prime \pm}}
$$

The last condition we have to require is the asymptotically conformally flat form of the new triad. This can be done by imposing

$$
e^{\prime 1}=\frac{r^{\prime} e^{\varphi^{\prime}}}{\sqrt{2}} d x^{\prime-}+O\left(r^{\prime-1}\right), \quad e^{\prime 2}=\frac{r^{\prime} e^{\varphi^{\prime}}}{\sqrt{2}} d x^{\prime+}+O\left(r^{\prime-1}\right) .
$$

The leading terms of $e_{\nu}^{\prime 1}=\Lambda_{a}^{1} e_{\mu}^{a} \frac{\partial x^{\mu}}{\partial x^{\prime \nu}}$ and $e_{\nu}^{\prime 2}=\Lambda_{a}^{2} e_{\mu}^{a} \frac{\partial x^{\mu}}{\partial x^{\prime \nu}}$ yield

$$
\frac{\partial x_{0}^{+}}{\partial x^{\prime-}}=0=\frac{\partial x_{0}^{-}}{\partial x^{\prime+}}=0, \quad e^{-E_{0}+r_{0}+\varphi} \frac{\partial x_{0}^{-}}{\partial x^{\prime-}}=e^{\varphi^{\prime}}=e^{E_{0}+r_{0}+\varphi} \frac{\partial x_{0}^{+}}{\partial x^{\prime+}} .
$$

Combining with equation (4.30), allows one to extract $A_{0}, B_{0}, E_{0}$ in terms of the other functions,

$$
E_{0}=\frac{1}{2} \ln \frac{\partial_{-}^{\prime} x_{0}^{-}}{\partial_{+}^{\prime} x_{0}^{+}}, \quad A_{0}=L \frac{e^{-\varphi-r_{0}}}{\sqrt{\partial_{-}^{\prime} x_{0}^{-} \partial_{+}^{\prime} x_{0}^{+}}} \frac{\partial r_{0}}{\partial x^{\prime+}}, \quad B_{0}=L \frac{e^{-\varphi-r_{0}}}{\sqrt{\partial_{-}^{\prime} x_{0}^{-} \partial_{+}^{\prime} x_{0}^{+}}} \frac{\partial r_{0}}{\partial x^{\prime-}} .
$$


It thus follows that the residual gauge symmetries are determined (i) by the change of variables $x^{ \pm}=x_{0}^{ \pm}\left(x^{\prime \pm}\right)$ at infinity, each depending on a single variable, which we assume to be orientation preserving $\partial_{+}^{\prime} x_{0}^{+}>0<\partial_{-}^{\prime} x_{0}^{-}$, and (ii) by $r_{0}\left(x^{\prime+}, x^{\prime-}\right)$.

For notational simplicity, we drop the subscript 0 on the change of variables at infinity and on the Weyl parameter in the next section.

\subsection{Action of conformal and Weyl group}

The group obtained in the previous section is the combined conformal and Weyl group and is parametrized by

$$
\left(x^{\prime+}\left(x^{+}\right), x^{\prime-}\left(x^{-}\right), r\left(x^{\prime+}, x^{-}\right)\right) .
$$

The last equation of (4.32) encodes the transformation law of $\varphi$,

$$
\varphi^{\prime}\left(x^{\prime+}, x^{\prime-}\right)=\varphi\left(x^{+}, x^{-}\right)+r\left(x^{\prime+}, x^{\prime-}\right)+\frac{1}{2} \ln \left(\partial_{+}^{\prime} x^{+} \partial_{-}^{\prime} x^{-}\right) .
$$

Note that, as a consequence, if $r\left(x^{\prime+}, x^{\prime-}\right), r^{s}\left(x^{\prime \prime+}, x^{\prime \prime-}\right)$ and $r^{c}\left(x^{\prime \prime+}, x^{\prime \prime-}\right)$ are associated to a first, a second successive and the combined transformation respectively, the composition law is

$$
r^{c}\left(x^{\prime \prime}, x^{\prime \prime-}\right)=r^{s}\left(x^{\prime \prime}, x^{\prime \prime-}\right)+r\left(x^{\prime+}, x^{\prime-}\right) .
$$

This group reduces to the conformal group for fixed conformal factor of the boundary metric: when $\varphi=\varphi^{\prime}$ it follows from (4.35) that $r$ is determined by the change of variables at infinity, $r=-\frac{1}{2} \ln \left(\partial_{+}^{\prime} x^{+} \partial_{-}^{\prime} x^{-}\right)$. When freezing the coordinate transformations, one remains with the additive group of Weyl rescalings that amount here to arbitrary shifts of $\varphi$.

As discussed in section 4.1, the on-shell metric, triads and spin connections are entirely determined by the arbitrary conformal factor $\varphi\left(x^{+}, x^{-}\right)$and the integration functions $C_{2}\left(x^{+}, x^{-}\right), C_{3}\left(x^{+}, x^{-}\right)$satisfying (4.18). To obtain the action of the group on the latter, we can extract the subleading terms of $l^{\prime}\left(x^{+}\right)=\Lambda_{1}^{b} e_{b}^{+}$and $n^{\prime}\left(x^{-}\right)=\Lambda_{2}^{b} e_{b}^{-}$. This gives

$$
\begin{aligned}
& C_{2}^{\prime}=e^{-2 r} \frac{\partial_{-}^{\prime} x^{-}}{\partial_{+}^{\prime} x^{+}} C_{2}+L^{2} e^{-2 \varphi^{\prime}}\left(\partial_{-}^{\prime 2} r+\left(\partial_{-}^{\prime} r\right)^{2}-2 \partial_{-}^{\prime} r \partial_{-}^{\prime} \varphi^{\prime}\right), \\
& C_{3}^{\prime}=e^{-2 r} \frac{\partial_{+}^{\prime} x^{+}}{\partial_{-}^{\prime} x^{-}} C_{3}+L^{2} e^{-2 \varphi^{\prime}}\left(\partial_{+}^{\prime 2} r+\left(\partial_{+}^{\prime} r\right)^{2}-2 \partial_{+}^{\prime} r \partial_{+}^{\prime} \varphi^{\prime}\right),
\end{aligned}
$$

which can also be written in terms of $\varphi$ using equation (4.35). Note that, by construction, the transformed $C_{2}^{\prime}\left(x^{\prime+}, x^{\prime-}\right), C_{3}\left(x^{\prime+}, x^{\prime-}\right)$ have to satisfy the transformed equations, i.e., equations (4.18) where all quantities, $C_{2}, C_{3}, \varphi, x^{ \pm}, \partial_{ \pm}$are primed.

In the particular case where $\varphi=0$, equations (4.18) reduce to $\partial_{+} C_{2 R}=0, \partial_{-} C_{3 R}=0$ so that $C_{2 R}=(8 \pi G L) T_{--}\left(x^{-}\right)$and $C_{3 R}=(8 \pi G L) T_{++}\left(x^{+}\right)$. Applying the particular Weyl transformation $x^{\prime \pm}=x^{ \pm}, r=\varphi^{\prime}$, and removing all primes, we obtain from (4.37) that the general solution to the dynamical equations (4.18) for arbitrary $\varphi$ is given by

$$
\begin{aligned}
& C_{2}=e^{-2 \varphi} L^{2}\left[\frac{8 \pi G}{L} T_{--}\left(x^{-}\right)+\partial_{-}^{2} \varphi-\left(\partial_{-} \varphi\right)^{2}\right], \\
& C_{3}=e^{-2 \varphi} L^{2}\left[\frac{8 \pi G}{L} T_{++}\left(x^{+}\right)+\partial_{+}^{2} \varphi-\left(\partial_{+} \varphi\right)^{2}\right] .
\end{aligned}
$$


Solution space can thus also be parametrized by the conformal factor $\varphi$ and the two integration functions $T_{ \pm \pm}\left(x^{ \pm}\right)$depending on a single variable each. The action of the asymptotic symmetry group on the latter can be extracted from equations (4.37),

$$
\begin{aligned}
T_{ \pm \pm}^{\prime}\left(x^{\prime \pm}\right) & =\left(\partial_{ \pm}^{\prime} x^{ \pm}\right)^{2} T_{ \pm \pm}\left(x_{0}^{ \pm}\right)-\frac{c_{ \pm}}{24 \pi}\left\{x^{ \pm}, x^{\prime \pm}\right\} \\
\Longleftrightarrow T_{ \pm \pm}^{\prime}\left(x^{\prime \pm}\right) & =\left(\partial_{ \pm} x^{\prime \pm}\right)^{-2}\left[T_{ \pm \pm}\left(x^{ \pm}\right)+\frac{c_{ \pm}}{24 \pi}\left\{x^{\prime \pm}, x^{ \pm}\right\}\right], \quad c_{ \pm}=\frac{3 L}{2 G},
\end{aligned}
$$

in terms of the Schwarzian derivative for a function $F$ of $x$,

$$
\{F, x\}=\partial_{x}^{2} \ln \partial_{x} F-\frac{1}{2}\left(\partial_{x} \ln \partial_{x} F\right)^{2},
$$

and with the characteristic values of the central charges for asymptotically $\mathrm{AdS}_{3}$ gravity [36]. In other words, the integration functions $T_{ \pm \pm}$are Weyl invariant, while under the centrally extended conformal group, one recovers the well-known coadjoint action, i.e., the standard transformation law of an energy-momentum tensor.

\section{$5 \quad 3 d$ asymptotically flat spacetimes at null infinity}

\subsection{Solution space}

The first gauge fixing conditions that we will assume are

$$
\kappa=\epsilon=\pi=0 \text {. }
$$

This is equivalent to $\Gamma_{a b 1}=0$ which can be achieved by a suitable Lorentz rotation. It implies that the tetrad is parallely transported along $l$ and that $l$ is the generator of an affinely parametrized null geodesic. In this case, $\nabla_{[a} l_{b]}=-2 l_{[a} m_{b]}(\tau-\beta)$, so that $l$ is always hypersurface orthornormal. It is a gradient if and only if

$$
\tau=\beta,
$$

a condition which will also be imposed in the following.

Introducing Bondi coordinates $x^{\mu}=(u, r, \phi), \mu=0,1,2$ such that the surfaces $u=c t e$ are null with normal vector $l, l_{\mu}=\delta_{\mu}^{0}$ and such that $r$ is the suitably normalized affine parameter on the null geodesics generated by $l$, the triad takes the form

$$
l=\frac{\partial}{\partial r}, \quad n=\frac{\partial}{\partial u}+W \frac{\partial}{\partial r}+V \frac{\partial}{\partial \phi}, \quad m=U \frac{\partial}{\partial r}+T \frac{\partial}{\partial \phi} .
$$

The associated cotriad is

$$
e^{1}=\left(-W+T^{-1} U V\right) d u+d r-T^{-1} U d \phi, \quad e^{2}=d u \quad e^{3}=-T^{-1} V d u+T^{-1} d \phi .
$$

Under these assumptions, the Newman-Penrose equations (A.1)-(A.6) fix the $r$ dependence of all spin coefficients according to

$$
\begin{aligned}
& \sigma=-\frac{1}{2} \frac{1}{r+C_{1}}, \quad \tau=\frac{C_{2}}{r+C_{1}}=\beta, \\
& \gamma=C_{3}-\frac{2 C_{2}^{2}}{r+C_{1}}, \quad \mu=\frac{C_{4}}{r+C_{1}}, \quad \nu=C_{5}-\frac{2 C_{2} C_{4}}{r+C_{1}} .
\end{aligned}
$$


for $C_{i}=C_{i}(u, \phi)$. When used in equations (A.10) and (A.11), the $r$ dependence of the triad is

$$
\begin{aligned}
T & =\frac{K_{1}}{r+C_{1}}, \quad V=-\frac{2 K_{1} C_{2}}{r+C_{1}}+K_{2}, \quad U=-C_{2}+\frac{K_{3}}{r+C_{1}}, \\
W & =-C_{3} r+K_{4}-\frac{2 C_{2} K_{3}}{r+C_{1}},
\end{aligned}
$$

with $K_{a}=K_{a}(u, \phi)$.

In order to solve the remaining equations, we will assume in addition that

$$
\sigma=-\frac{1}{2 r}+O\left(r^{-3}\right), \quad \tau=O\left(r^{-2}\right), \quad V=O\left(r^{-1}\right) .
$$

The first condition can be satsified by changing the affine parameter $r \rightarrow r+C_{1}$. We can then do a Lorentz transformation with $a=d=1, c=0$ and $b=C_{2}$ in order to impose $C_{2}=0$, and finally a change of coordinates $\partial_{u} \phi^{\prime}=-K_{2} \partial_{\phi} \phi^{\prime}$ to obtain $K_{2}=0$. Note however that both of these last two transformations are only valid asymptotically. Requiring them to preserve the gauge fixing conditions will require subleading terms in a similar way as in the computation of section 5.2. On the level of solutions, the additional conditions simply amount to setting

$$
C_{1}=C_{2}=K_{2}=0
$$

Redefining $K_{1}=e^{-\varphi}$, the remaining equations, i.e., (A.7)-(A.9) and (A.12), are equivalent to

$$
C_{4}=\frac{1}{2} K_{4}, \quad C_{3}=\partial_{u} \varphi, \quad C_{5}=e^{-\varphi} \partial_{u} \partial_{\phi} \varphi
$$

where

$$
\begin{aligned}
\partial_{u} K_{4}+2 \partial_{u} \varphi K_{4} & =2 e^{-2 \varphi}\left(\partial_{u} \partial_{\phi}^{2} \varphi-\partial_{\phi} \varphi \partial_{u} \partial_{\phi} \varphi\right), \\
\partial_{u} K_{3}+2 \partial_{u} \varphi K_{3} & =e^{-\varphi} \partial_{\phi} K_{4} .
\end{aligned}
$$

These equations can be integrated directly, but we will again generate the solution by using the asymptotic symmetry group below.

In this case, (5.5) and (5.6) simplify to

$$
\begin{aligned}
& \sigma=-\frac{1}{2 r}, \quad \tau=\beta=0, \quad \gamma=\partial_{u} \varphi, \quad \mu=\frac{K_{4}}{2 r}, \quad \nu=e^{-\varphi} \partial_{u} \partial_{\phi} \varphi, \\
& T=\frac{e^{-\varphi}}{r}, \quad W=-\partial_{u} \varphi r+K_{4}, \quad U=\frac{K_{3}}{r}, \quad V=0 .
\end{aligned}
$$

\subsection{Residual gauge symmetries}

The residual gauge symmetries again consist of the subset of gauge transformations that preserve the set of conditions determining the asymptotic solution space. We will consider a general change of coordinates of the form

$$
u=u\left(u^{\prime}, r^{\prime}, \phi^{\prime}\right), \quad r=r\left(u^{\prime}, r^{\prime}, \phi^{\prime}\right), \quad \phi=\phi\left(u^{\prime}, r^{\prime}, \phi^{\prime}\right),
$$


combined with an arbitrary local Lorentz transformation. The unknowns are $A, B, E, u, r, \phi$ as functions of $u^{\prime}, r^{\prime}, \phi^{\prime}$.

Using the $a=1$ component of the transformation law for the triad

$$
e_{a}^{\prime \mu} \frac{\partial x^{\nu}}{\partial x^{\prime \mu}}=\Lambda_{a}^{b} e_{b}^{\nu}
$$

it follows that imposing $l^{\prime \mu}=\delta_{r^{\prime}}^{\mu}$ is equivalent to the radial equations,

$$
\begin{aligned}
& \frac{\partial u}{\partial r^{\prime}}=B^{2} e^{-E}, \\
& \frac{\partial \phi}{\partial r^{\prime}}=2 B(1+A B) T, \\
& \frac{\partial r}{\partial r^{\prime}}=(1+A B)^{2} e^{E}+B^{2} e^{-E} W+2 B(1+A B) U .
\end{aligned}
$$

The gauge fixing on the new spin coefficients takes the form $\breve{\omega}_{1}^{\prime}=0$. The component $a=1$ of the last equation of (3.19) can be rewritten as

$$
g^{-1} \partial_{r^{\prime}} g=\Lambda_{1}^{b} \breve{\omega}_{b} .
$$

This is equivalent to three conditions on the rotation parameters, which can be suitably combined to yield

$$
\begin{aligned}
& \frac{\partial B}{\partial r^{\prime}}=2 B(1+A B) e^{E} \sigma, \\
& \frac{\partial A}{\partial r^{\prime}}=-B^{2} e^{-2 E} \nu-2 B(1+A B) e^{-E} \mu+2 A^{2} B(1+A B) e^{E} \sigma, \\
& \frac{\partial E}{\partial r^{\prime}}=-B^{2} e^{-E} \gamma-4 A B(1+A B) e^{E} \sigma .
\end{aligned}
$$

The set of equations (5.14) and (5.16) forms a system of differential equations for the radial dependence of the unknown functions. In order to solve it asymptotically, we assume that the functions have the following asymptotic behavior,

$$
r=O\left(r^{\prime}\right), \quad A, E, u, \phi=O(1), \quad B=O\left(r^{-1}\right) .
$$

The unknown $r$ can be traded for $\chi=r e^{-E}=O\left(r^{\prime}\right)$ satisfying

$$
\frac{\partial \chi}{\partial r^{\prime}}=1-A^{2} B^{2}+B^{2} e^{-2 E} K_{4}+\frac{2 B}{\chi}(1+A B) e^{-2 E} K_{3}=1+O\left(r^{\prime-2}\right) .
$$

The solution is given by

$$
\chi=r^{\prime}+\chi_{0}(u, \phi)+O\left(r^{\prime-1}\right),
$$

which, when introduced into the other radial equations, gives

$$
\begin{aligned}
B & =B_{0}(u, \phi) r^{\prime-1}+\left(A_{0} B_{0}^{2}-B_{0} \chi_{0}\right) r^{\prime-2}+O\left(r^{\prime-3}\right), \\
A & =A_{0}(u, \phi)+\left(B_{0}^{2} e^{-2 E_{0}-\varphi} \partial_{u} \partial_{\phi} \varphi+B_{0} K_{4} e^{-2 E_{0}}+A_{0}^{2} B_{0}\right) r^{\prime-1}+O\left(r^{\prime-2}\right), \\
E & =E_{0}(u, \phi)+\left(B_{0}^{2} e^{-E_{0}} \partial_{u} \varphi-2 A_{0} B_{0}\right) r^{\prime-1}+O\left(r^{\prime-2}\right), \\
u & =u_{0}(u, \phi)-B_{0}^{2} e^{-E_{0}} r^{\prime-1}+O\left(r^{\prime-2}\right), \\
\phi & =\phi_{0}(u, \phi)-2 B_{0} e^{-\varphi-E_{0}} r^{\prime-1}+O\left(r^{\prime-2}\right) .
\end{aligned}
$$


At this stage, we have fixed the radial dependence of all the unknowns and are left with six functions $A_{0}, B_{0}, E_{0}, u_{0}, \phi_{0}, \chi_{0}$ of $u^{\prime}$ and $\phi^{\prime}$.

We now have to require $l_{\mu}^{\prime}=\delta_{\mu}^{u^{\prime}}$. After having imposed $l^{\prime}=\frac{\partial}{\partial r^{\prime}}$, one has in particular that $l_{r^{\prime}}^{\prime}=0$. This follows from the combination of $l_{r^{\prime}}^{\prime}=\Lambda_{b}^{2} e^{b}{ }_{\nu} \frac{\partial x^{\nu}}{\partial r^{\prime}}$ and $\frac{\partial x^{\nu}}{\partial r^{\prime}}=\Lambda_{1}{ }^{c} e_{c}{ }^{\nu}$. For the remaining components of $l_{\mu}^{\prime}$ it is enough to verify that $l_{u^{\prime}}^{\prime}=1+o\left(r^{\prime 0}\right), l_{\phi}^{\prime}=o\left(r^{\prime 0}\right)$ since the equation of motion $d e^{a}+\Gamma^{\prime a}{ }_{b} e^{b}=0$ for $a=2$ implies $\partial_{r^{\prime}} l_{\nu^{\prime}}-\partial_{\nu^{\prime}} l_{\mu^{\prime}}^{\prime} e_{1} \mu^{\prime}+\Gamma_{1 b 1}^{\prime} e^{\prime b} \nu^{\prime}-$ $\Gamma_{11 \nu^{\prime}}^{\prime}=0$. This reduces to $\partial_{r^{\prime}} l_{\nu^{\prime}}^{\prime}=\partial_{\nu^{\prime}} l_{r^{\prime}}^{\prime}$, and thus to $\partial_{r^{\prime}} l_{u^{\prime}}^{\prime}=0=\partial_{r^{\prime}} l_{\phi}^{\prime}$. Extracting the leading order from

$$
e_{\mu}^{\prime 2}=\left(-c^{2} W+d^{2}\right) \frac{\partial u}{\partial x^{\prime \mu}}+c^{2} \frac{\partial r}{\partial x^{\prime \mu}}+\left(-c^{2} U+c d\right) \frac{1}{T} \frac{\partial \phi}{\partial x^{\prime \mu}}
$$

we get

$$
e^{-E_{0}}=\frac{\partial u_{0}}{\partial u^{\prime}}-B_{0} e^{\varphi} \frac{\partial \phi_{0}}{\partial u^{\prime}}, \quad 0=\frac{\partial u_{0}}{\partial \phi^{\prime}}-B_{0} e^{\varphi} \frac{\partial \phi_{0}}{\partial \phi^{\prime}} .
$$

We still have to impose three conditions: $V^{\prime}=O\left(r^{\prime-1}\right), \sigma^{\prime}=-\frac{1}{2 r^{\prime}}+O\left(r^{\prime-3}\right), \tau^{\prime}=O\left(r^{\prime-2}\right)$. The first one is a condition on the triad and can be imposed by requiring $e_{u}^{\prime 3}=O(1)$. More generally, we have

$$
e_{\mu}^{\prime 3}=(-2 a c W+2 b d) \frac{\partial u}{\partial x^{\prime \mu}}+2 a c \frac{\partial r}{\partial x^{\prime \mu}}+(-2 a c U+a d+b c) \frac{1}{T} \frac{\partial \phi}{\partial x^{\prime \mu}},
$$

and, requiring the new cotriads to have the same form in the new coordinate system than they had in the old one, the leading terms of $e_{u}^{\prime 3}$ and $e_{\phi}^{\prime 3}$ yield

$$
0=e^{\varphi} \frac{\partial \phi_{0}}{\partial u^{\prime}}, \quad e^{-E_{0}+\varphi^{\prime}}=e^{\varphi} \frac{\partial \phi_{0}}{\partial \phi^{\prime}} .
$$

Note in particular that our choice of parametrization for the Lorentz rotations leads to $\frac{\partial \phi_{0}}{\partial \phi^{\prime}}>0$. The first equation is equivalent to $V^{\prime}=O\left(r^{\prime-1}\right)$ while the second one gives the transformation law of $\varphi$. Combining (5.23) with (5.21), we obtain

$$
\frac{\partial \phi_{0}}{\partial u^{\prime}}=0, \quad \frac{\partial u_{0}}{\partial u^{\prime}}=e^{-E_{0}}, \quad B_{0}=e^{-\varphi}\left(\frac{\partial \phi_{0}}{\partial \phi^{\prime}}\right)^{-1} \frac{\partial u_{0}}{\partial \phi^{\prime}}, \quad e^{\varphi^{\prime}}=e^{E_{0}+\varphi} \frac{\partial \phi_{0}}{\partial \phi^{\prime}} .
$$

To implement the last two conditions, we will use the transformation law of $\breve{\omega}_{3}$ given in the last equation of (3.19). Imposing $\left(\breve{\omega}_{3}^{\prime}\right)_{11}=O\left(r^{\prime-2}\right)$ and $\left(\breve{\omega}_{3}^{\prime}\right)_{21}=\frac{1}{2 r^{\prime}}+O\left(r^{\prime-3}\right)$, we get

$$
\begin{aligned}
& \frac{1}{2 r^{\prime}}\left(B_{0} e^{-E_{0}} \partial_{u} \varphi-A_{0}\right)+\frac{1}{2} \delta^{\prime} E+B \delta^{\prime} A=O\left(r^{\prime-2}\right), \\
& \frac{1}{r^{\prime 2}}\left(-\frac{1}{2} \chi_{0}+2 A_{0} B_{0}-B_{0}^{2} e^{-E_{0}} \gamma\right) \\
& \quad-B^{2} \delta^{\prime} A-B(1+A B) \delta^{\prime} E+\delta^{\prime} B=O\left(r^{\prime-3}\right) .
\end{aligned}
$$

From the general solution, we have $\delta^{\prime}=U^{\prime} \partial_{r}^{\prime}+T^{\prime} \partial_{\phi}^{\prime}$ where $U^{\prime}=O(1)$ and $T^{\prime}=e^{-\varphi^{\prime}} r^{\prime-1}+$ $O\left(r^{\prime-2}\right)$. Inserting this into the two equations we can extract the value of $A_{0}$ and $\chi_{0}$,

$$
A_{0}=e^{-\varphi^{\prime}} \frac{\partial E_{0}}{\partial \phi^{\prime}}+B_{0} e^{-E_{0}} \frac{\partial \varphi}{\partial u}, \quad \chi_{0}=2 A_{0} B_{0}+2 e^{-\varphi^{\prime}} \frac{\partial B_{0}}{\partial \phi^{\prime}} .
$$


The asymptotic symmetry group is thus parametrised by three functions $u_{0}\left(u^{\prime}, \phi^{\prime}\right)$, $\phi_{0}\left(u^{\prime}, \phi^{\prime}\right)$ and $E_{0}\left(u^{\prime}, \phi^{\prime}\right)$ satisfying the constraints

$$
\frac{\partial \phi_{0}}{\partial u^{\prime}}=0, \quad \frac{\partial u_{0}}{\partial u^{\prime}}=e^{-E_{0}} .
$$

Note that, when taking these into account, the Jacobian matrices for the change of coordinates at infinity are

$$
\left(\begin{array}{cc}
\frac{\partial u_{0}}{\partial u^{\prime}}=e^{-E_{0}} & \frac{\partial u_{0}}{\partial \phi^{\prime}} \\
\frac{\partial \phi_{0}}{\partial u^{\prime}}=0 & \frac{\partial \phi_{0}}{\partial \phi^{\prime}}=e^{\varphi^{\prime}-\varphi-E_{0}}
\end{array}\right),\left(\begin{array}{cc}
\frac{\partial u_{0}^{\prime}}{\partial u}=e^{E_{0}} & \frac{\partial u_{0}^{\prime}}{\partial \phi}=-e^{2 E_{0}-\varphi^{\prime}+\varphi} \frac{\partial u_{0}}{\partial \phi^{\prime}} \\
\frac{\partial \phi_{0}^{\prime}}{\partial u}=0 & \frac{\partial \phi_{0}^{\prime}}{\partial \phi}=e^{E_{0}-\varphi^{\prime}+\varphi}
\end{array}\right)
$$

For notational simplicity, we will drop the subscript 0 on the functions determining the change of coordinates at infinity and on the Weyl parameter in the next two section.

\subsection{Combined BMS3 and Weyl group}

From equation (5.27), it follows that $E\left(u^{\prime}, \phi^{\prime}\right)$ is determined by the function $u\left(u^{\prime}, \phi^{\prime}\right)$ and, conversely, that the knowledge of such a function $E$ allows one to recover the complete change of coordinates, up to an arbitrary function $\hat{u}^{\prime}\left(\phi^{\prime}\right)$,

$$
u\left(u^{\prime}, \phi^{\prime}\right)=\int_{\hat{u}^{\prime}}^{u^{\prime}} d v^{\prime} e^{-E} .
$$

Note that the point with coordinates $\left(\hat{u}^{\prime}\left(\phi^{\prime}\right), \phi^{\prime}\right)$ in the new coordinate system is described by $(0, \phi)$ in the original coordinate system. When considering the inverse transformation, we can write,

$$
u^{\prime}(u, \phi)=\int_{\hat{u}}^{u} d v e^{E}
$$

where $E_{0}$ is now considered as a function of the original coordinate system through $E_{0}\left(u^{\prime}(u, \phi), \phi^{\prime}(u, \phi)\right)$ and the point with coordinates $(\hat{u}(\phi), \phi)$ is described by $\left(0, \phi^{\prime}\right)$ in the new coordinate system.

Equation (5.24) is equivalent to the transformation law of field $\varphi$,

$$
\varphi^{\prime}\left(u^{\prime}, \phi^{\prime}\right)=\varphi(u, \phi)+E\left(u^{\prime}, \phi^{\prime}\right)+\ln \frac{\partial \phi}{\partial \phi^{\prime}} .
$$

This can be used to trade $\hat{u}(\phi)$ for

$$
\beta(\phi)=\int_{\hat{u}}^{0} d v e^{-\varphi(v, \phi)},
$$

which can be inverted since the integrand is positive.

The combined $\mathrm{BMS}_{3}$ and Weyl group can be parametrized by

$$
\left(\phi_{0}^{\prime}(\phi), \beta(\phi), E\left(u^{\prime}, \phi^{\prime}\right)\right) \text {. }
$$

Note that the transformation law of

$$
\widetilde{u}(u, \phi)=\int_{0}^{u} d v e^{-\varphi(v, \phi)},
$$


is

$$
\widetilde{u}^{\prime}\left(u^{\prime}, \phi^{\prime}\right)=\frac{\partial \phi^{\prime}}{\partial \phi}[\widetilde{u}(u, \phi)+\beta(\phi)]
$$

In particular, if

$$
\beta(\phi), E\left(u^{\prime}, \phi^{\prime}\right), \quad \beta^{s}\left(\phi^{\prime}\right), E^{s}\left(u^{\prime \prime}, \phi^{\prime \prime}\right), \quad \beta^{c}(\phi), E^{c}\left(u^{\prime \prime}, \phi^{\prime \prime}\right),
$$

are associated to a first, a second successive and their combined transformation respectively, equations (5.31) and (5.34) imply that

$$
\begin{aligned}
\beta^{c}(\phi) & =\frac{\partial \phi}{\partial \phi^{\prime}} \beta^{s}\left(\phi^{\prime}\right)+\beta(\phi), \\
E^{c}\left(u^{\prime \prime}, \phi^{\prime \prime}\right) & =E^{s}\left(u^{\prime \prime}, \phi^{\prime \prime}\right)+E\left(u^{\prime}, \phi^{\prime}\right) .
\end{aligned}
$$

For fixed diffeomorphism on the circle, $\phi^{\prime}=\phi$, the first of (5.36) describes the abelian subgroup of supertranslations, while, if in addition one restricts to the subgroup without supertranslations, i.e., when all $\beta$ 's vanish, so do the $\hat{u}$ 's and $u$ is unchanged. The second of (5.36) then describes the abelian subgroup of Weyl rescalings.

Alternatively, one can define

$$
\mathcal{U}(u, \phi)=e^{\varphi} \widetilde{u}, \quad \alpha(u, \phi)=e^{\varphi} \beta,
$$

and parametrize the combined $\mathrm{BMS}_{3}$ and Weyl group by

$$
\left(\phi^{\prime}(\phi), \alpha(u, \phi), E\left(u^{\prime}, \phi^{\prime}\right)\right) \text {. }
$$

In this case, equation (5.35) and the first of equation (5.36) are replaced by

$$
\begin{aligned}
\mathcal{U}^{\prime}\left(u^{\prime}, \phi^{\prime}\right) & =e^{E\left(u^{\prime}, \phi^{\prime}\right)}[\mathcal{U}(u, \phi)+\alpha(u, \phi)], \\
\alpha^{c}(u, \phi) & =e^{-E\left(u^{\prime}, \phi^{\prime}\right)} \alpha^{s}\left(u^{\prime}, \phi^{\prime}\right)+\alpha(u, \phi) .
\end{aligned}
$$

Note that if $\varphi$ does not depend on $u$ then $\tilde{\mathcal{U}}=e^{-\varphi} u, \beta=-e^{-\varphi} \hat{u}(\phi)$ whereas $\mathcal{U}=u$ and $\alpha(\phi)=-\hat{u}$. If furthermore $\varphi^{\prime}$ does not depend on $u^{\prime}$, then neither does $E$ and $u^{\prime}(u, \phi)=e^{E\left(\phi^{\prime}\right)}(u+\alpha)$. The standard definition of the $\mathrm{BMS}_{3}$ group is then recovered when the conformal factor is fixed to be zero, i.e., when $\varphi(u, \phi)=0=\varphi^{\prime}\left(u^{\prime}, \phi^{\prime}\right)$, in which case it follows from equation (5.31) that the Weyl transformations are frozen to $e^{E_{F}}=\frac{\partial \phi^{\prime}}{\partial \phi}$.

\subsection{Action on solution space}

Solution space is parametrized by the three functions $\varphi, K_{3}, K_{4}$ satisfying the evolution equations (5.10). The action of the group on the conformal factor $\varphi$ has already been computed in the previous section. We can extract the transformation law of $K_{4}$ from $\left(\breve{\omega}_{3}^{\prime}\right)_{12}$ and the one of $K_{3}$ from the second order of $e_{\phi}^{\prime 1}$,

$$
\begin{aligned}
K_{4}^{\prime}= & e^{-2 E} K_{4}+A_{0}^{2}+2 e^{-\varphi^{\prime}} \partial_{\phi^{\prime}} A_{0}+2 B_{0} e^{-2 E-\varphi} \partial_{\phi} \partial_{u} \varphi \\
K_{3}^{\prime}= & e^{-2 E} K_{3}+2 e^{-2 E} B_{0} K_{4}-2 e^{-\varphi^{\prime}} \partial_{\phi^{\prime}}\left(e^{-\varphi^{\prime}} \partial_{\phi^{\prime}} B_{0}\right) \\
& -2 A_{0} e^{-\varphi^{\prime}} \partial_{\phi^{\prime}} B_{0}+2 B_{0}^{2} e^{-2 E-\varphi} \partial_{\phi} \partial_{u} \varphi .
\end{aligned}
$$


By construction, the transformed quantities have to satisfy the transformed equations, i.e., equations (5.10) where all quantities, $K_{3}, K_{4}, \varphi, u, \phi, \partial_{u}, \partial_{\phi}$ are primed.

In the particular case where $\varphi=0$, equations (5.10) reduce to $\partial_{u} K_{4 R}=0, \partial_{u} K_{3 R}=$ $\partial_{\phi} K_{4 R}$, so that $K_{4 R}=(16 \pi G) p(\phi), K_{3 R}=(16 \pi G)\left(j(\phi)+u \partial_{\phi} p\right)$. Applying the particular Weyl transformation $\phi^{\prime}=\phi, u^{\prime}=\int_{0}^{u} d v e^{E}, E\left(u^{\prime}, \phi^{\prime}\right)=\varphi^{\prime}\left(u^{\prime}, \phi^{\prime}\right)$, with inverse transformation $u=\int_{0}^{u^{\prime}} d v^{\prime} e^{-\varphi^{\prime}\left(v^{\prime}, \phi^{\prime}\right)}$, we obtain from (5.40) that

$$
K_{4}^{\prime}=e^{-2 \varphi^{\prime}}\left[K_{4 R}+2 \partial_{\phi^{\prime}}^{2} \varphi^{\prime}-\left(\partial_{\phi^{\prime}} \varphi^{\prime}\right)^{2}\right], K_{3}^{\prime}=e^{-2 \varphi^{\prime}}\left[K_{3 R}+2 \partial_{\phi^{\prime}} u K_{4 R}-2 \partial_{\phi^{\prime}}^{3} u\right] .
$$

After removing all primes and writing the inverse transformation as in (5.34), it follows that the general solution to the dynamical equations (5.10) for arbitray $\varphi$ is given by

$$
\begin{aligned}
& K_{4}=(16 \pi G) e^{-2 \varphi}\left[p(\phi)+\frac{1}{16 \pi G}\left(2 \partial_{\phi}^{2} \varphi-\left(\partial_{\phi} \varphi\right)^{2}\right)\right], \\
& K_{3}=(16 \pi G) e^{-2 \varphi}\left[j(\phi)+\widetilde{u} \partial_{\phi} p(\phi)+2 \partial_{\phi} \widetilde{u} p(\phi)-\frac{1}{8 \pi G} \partial_{\phi}^{3} \widetilde{u}\right] .
\end{aligned}
$$

The final parametrisation of the solution space studied in section 5.1 is given by the conformal factor $\varphi$ and the two functions $p(\phi)$ and $j(\phi)$. Their transformation laws under the combined $\mathrm{BMS}_{3}$ and Weyl group is given by

$$
\begin{aligned}
p^{\prime}\left(\phi^{\prime}\right) & =\left(\frac{\partial \phi}{\partial \phi^{\prime}}\right)^{2}\left[p(\phi)+\frac{c_{2}}{24 \pi}\left\{\phi^{\prime}, \phi\right\}\right], \quad c_{1}=0, \quad c_{2}=\frac{3}{G}, \\
j^{\prime}\left(\phi^{\prime}\right) & =\left(\frac{\partial \phi}{\partial \phi^{\prime}}\right)^{2}\left[j(\phi)-2 p(\phi) \partial_{\phi} \beta-\partial_{\phi} p \beta+\frac{c_{2}}{24 \pi} \partial_{\phi}^{3} \beta+\frac{c_{1}}{24 \pi}\left\{\phi^{\prime}, \phi\right\}\right] .
\end{aligned}
$$

The central charges have the characteristic values for asymptotically flat three-dimensional Einstein gravity [37]. These quantities are thus Weyl invariant, which needs to be the case by construction since a Weyl transformation applied to $K_{3}, K_{4}$ amounts to applying the combined Weyl transformation to $p, j$ with the associated change of $\widetilde{u}$. Their transformations under the $\mathrm{BMS}_{3}$ group agree with those derived by different methods in [22, 38].

\section{$6 \quad 4 d$ asymptotically flat spacetimes at null infinity}

\subsection{Newman-Penrose formalism in $4 \mathrm{~d}$}

In four dimensions with signature $(+,-,-,-)$, we use

$$
\eta_{a b}=\left(\begin{array}{cccc}
0 & 1 & 0 & 0 \\
1 & 0 & 0 & 0 \\
0 & 0 & 0 & -1 \\
0 & 0 & -1 & 0
\end{array}\right) .
$$

The different elements of the null tetrad are denoted by $e_{a}=(l, n, m, \bar{m})$, with the associated directional covariant derivatives denoted by $(D, \Delta, \delta, \bar{\delta})$. In particular,

$$
g^{\mu \nu}=l^{\mu} n^{\nu}+l^{\nu} n^{\mu}-m^{\mu} \bar{m}^{\nu}-m^{\nu} \bar{m}^{\mu}, \quad \nabla_{a}=n_{a} D+l_{a} \Delta-m_{a} \bar{\delta}-\bar{m}_{a} \delta .
$$


The 24 independent $\Gamma_{a b c}$ 's are parametrized through 12 complex scalars,

\begin{tabular}{|c|c|c|c|}
\hline$\nabla$ & $m^{a} \nabla l_{a}$ & $\frac{1}{2}\left(n^{a} \nabla l_{a}-\bar{m}^{a} \nabla m_{a}\right)$ & $-\bar{m}^{a} \nabla n_{a}$ \\
\hline$D$ & $\kappa=\Gamma_{311}$ & $\epsilon=\frac{1}{2}\left(\Gamma_{211}-\Gamma_{431}\right)$ & $\pi=-\Gamma_{421}$ \\
\hline$\Delta$ & $\tau=\Gamma_{312}$ & $\gamma=\frac{1}{2}\left(\Gamma_{212}-\Gamma_{432}\right)$ & $\nu=-\Gamma_{422}$ \\
\hline$\delta$ & $\sigma=\Gamma_{313}$ & $\beta=\frac{1}{2}\left(\Gamma_{213}-\Gamma_{433}\right)$ & $\mu=-\Gamma_{423}$ \\
\hline $\bar{\delta}$ & $\rho=\Gamma_{314}$ & $\alpha=\frac{1}{2}\left(\Gamma_{214}-\Gamma_{434}\right)$ & $\lambda=-\Gamma_{424}$ \\
\hline
\end{tabular}

where the associated complex conjugates are obtained by exchanging the indices 3 and 4 .

In order to describe Lorentz transformations in four dimensions in terms of a null tetrad, one associates to a real vector $v=v^{a} e_{a}$, with $v^{1}, v^{2} \in \mathbb{R}, v^{4}=\overline{v^{3}} \in \mathbb{C}$, a $2 \times 2$ hermitian matrix $\widehat{v}=v^{a} \widehat{j}_{a}$, where the $\widehat{j}_{a}$ are chosen as

$$
\widehat{j}_{1}=\left(\begin{array}{ll}
1 & 0 \\
0 & 0
\end{array}\right), \widehat{j}_{2}=\left(\begin{array}{ll}
0 & 0 \\
0 & 1
\end{array}\right), \widehat{j}_{3}=\left(\begin{array}{ll}
0 & 1 \\
0 & 0
\end{array}\right), \widehat{j}_{4}=\left(\begin{array}{ll}
0 & 0 \\
1 & 0
\end{array}\right) .
$$

In this case

$$
\operatorname{det} \widehat{v}=\frac{1}{2} \eta_{a b} v^{a} v^{b}, \widehat{j}_{b}^{T} \epsilon \widehat{j}_{a}+\widehat{j}_{a}^{T} \epsilon \widehat{j}_{b}=\eta_{a b} \epsilon, \widehat{j}_{b} \epsilon \widehat{j}_{a}^{T}+\widehat{j}_{a} \epsilon \widehat{j}_{b}^{T}=\eta_{a b} \epsilon, \widehat{j}^{a}\left(\widehat{j}_{b}+\widehat{j}_{b}^{T}\right) \widehat{j}_{a}^{T}=0
$$

where

$$
\epsilon=\left(\begin{array}{cc}
0 & 1 \\
-1 & 0
\end{array}\right)
$$

For an element $g \in \mathrm{SL}(2, \mathbb{C})$, one considers the transformation

$$
\widehat{g}_{a} g^{\dagger} v^{a}=\widehat{j}_{a} \Lambda_{b}^{a} v^{b}, \quad \epsilon g^{T}=g^{-1} \epsilon .
$$

More explicitly, if

$$
g=\left(\begin{array}{ll}
a & b \\
c & d
\end{array}\right)
$$

with $a d-b c=1$ and $a, b, c, d \in \mathbb{C}$, then

$$
\Lambda_{b}^{a}=\left(\begin{array}{llll}
a \bar{a} & b \bar{b} & a \bar{b} & b \bar{a} \\
c \bar{c} & d \bar{d} & c \bar{d} & d \bar{c} \\
a \bar{c} & b \bar{d} & a \bar{d} & b \bar{c} \\
c \bar{a} & d \bar{b} & c \bar{b} & d \bar{a}
\end{array}\right), \Lambda_{a}{ }^{b}=\left(\begin{array}{cccc}
d \bar{d} & c \bar{c} & -d \bar{c} & -c \bar{d} \\
b \bar{b} & a \bar{a} & -b \bar{a} & -a \bar{b} \\
-d \bar{b} & -c \bar{a} & d \bar{a} & c \bar{b} \\
-b \bar{d} & -a \bar{c} & b \bar{c} & a \bar{d}
\end{array}\right),
$$

where the first index is the lign index.

The standard three classes of rotations [30] are then given by

- class I for which $l^{\prime}=l, m^{\prime}=m+A l, \bar{m}^{\prime}=\bar{m}+\bar{A} l, n^{\prime}=n+\bar{A} m+A \bar{m}+A \bar{A} l$ : $a=1=d, c=0, b=-\bar{A}, A \in \mathbb{C}$,

- class II for which $n^{\prime}=n, m^{\prime}=m+B n, \bar{m}^{\prime}=\bar{m}+\bar{B} n, l^{\prime}=l+\bar{B} m+B \bar{m}+B \bar{B} n$ : $a=1=d, b=0, c=-B, B \in \mathbb{C}$, 
- class III for which $l^{\prime}=e^{-E_{R}} l, n^{\prime}=e^{E_{R}} n, m^{\prime}=e^{i E_{I}} m, \bar{m}^{\prime}=e^{-i E_{I}} \bar{m}$ :

$a=e^{-E / 2}, d=e^{E / 2}, b=0=c, E=E_{R}+i E_{I} \in \mathbb{C}$.

Finally, the $\mathrm{SL}(2, \mathbb{C})$ group element corresponding to a combined rotation $I I \circ I \circ I I I$ is given by

$$
g=\left(\begin{array}{cc}
e^{-E / 2} & -\bar{A} e^{E / 2} \\
-B e^{-E / 2} & (1+\bar{A} B) e^{E / 2}
\end{array}\right)
$$

Defining

$$
\widehat{\omega}=-\frac{1}{2} \widehat{j}_{a} \widehat{\epsilon} \hat{j}_{b}^{T} \Gamma^{a b}=\left(\begin{array}{cc}
-\Gamma_{42} & -\frac{1}{2}\left(\Gamma_{21}-\Gamma_{43}\right) \\
-\frac{1}{2}\left(\Gamma_{21}-\Gamma_{43}\right) & \Gamma_{31}
\end{array}\right),
$$

the transformation law of Lorentz connection becomes

$$
\widehat{\omega}^{\prime}=g \widehat{\omega} g^{T}-g \epsilon d g^{T} .
$$

More explicitly, for the spin coefficients encoded in

$$
\widehat{\omega}_{1}=\left(\begin{array}{cc}
\pi & -\epsilon \\
-\epsilon & \kappa
\end{array}\right), \widehat{\omega}_{2}=\left(\begin{array}{cc}
\nu & -\gamma \\
-\gamma & \tau
\end{array}\right), \widehat{\omega}_{3}=\left(\begin{array}{cc}
\mu & -\beta \\
-\beta & \sigma
\end{array}\right), \widehat{\omega}_{4}=\left(\begin{array}{cc}
\lambda & -\alpha \\
-\alpha & \rho
\end{array}\right),
$$

one finds

$$
\widehat{\omega}_{a}^{\prime}=\Lambda_{a}^{c} g \widehat{\omega}_{c} g^{T}-g \epsilon e_{a}^{\prime}\left(g^{T}\right) .
$$

Alternatively, one can use $\breve{v}=\widehat{v} \epsilon$ in order to describe real vectors. The associated basis is

$$
\breve{j}_{1}=\left(\begin{array}{ll}
0 & 1 \\
0 & 0
\end{array}\right), \breve{j}_{2}=\left(\begin{array}{cc}
0 & 0 \\
-1 & 0
\end{array}\right), \breve{j}_{3}=\left(\begin{array}{cc}
-1 & 0 \\
0 & 0
\end{array}\right), \breve{j}_{4}=\left(\begin{array}{ll}
0 & 0 \\
0 & 1
\end{array}\right),
$$

so that

$$
\operatorname{det} \breve{v}=\frac{1}{2} \eta_{a b} v^{a} v^{b}, \breve{j}_{b}^{T} \epsilon \breve{j}_{a}+\breve{j}_{a}^{T} \epsilon \breve{j}_{b}=\eta_{a b} \epsilon, \check{j}_{b} \epsilon \breve{j}_{a}^{T}+\breve{j}_{a} \epsilon \breve{j}_{b}^{T}=\eta_{a b} \epsilon, \breve{j}^{a}\left(\check{j}_{b}+\breve{j}_{b}^{T}\right) \check{j}_{a}^{T}=0 .
$$

In this case, we have

$$
g \breve{j}_{a} g^{-1} v^{a}=\breve{j}_{a} \Lambda_{b}^{a} v^{b}, \quad \breve{\omega}^{\prime}=g \breve{\omega}^{-1}-d g g^{-1}, \quad \breve{\omega}_{a}^{\prime}=\Lambda_{a}^{c} g \breve{\omega}_{c} g^{-1}-e_{a}^{\prime}(g) g^{-1},
$$

where

$$
\breve{\omega}_{1}=\left(\begin{array}{cc}
\epsilon & \pi \\
-\kappa & -\epsilon
\end{array}\right), \breve{\omega}_{2}=\left(\begin{array}{cc}
\gamma & \nu \\
-\tau & -\gamma
\end{array}\right), \breve{\omega}_{3}=\left(\begin{array}{cc}
\beta & \mu \\
-\sigma & -\beta
\end{array}\right), \breve{\omega}_{4}=\left(\begin{array}{cc}
\alpha & \lambda \\
-\rho & -\alpha
\end{array}\right) .
$$

For the Weyl scalars, we follow the conventions of [33, 39], which differ by a sign from those of [30] and those of [25, 27] (when taking into account in addition the correction for $\Psi_{2}$ given in $\left.[26,29]\right)$. If $C_{a b c d}$ denote the components of the Weyl tensor and $\Psi_{A B C D}$ the associated Weyl spinor,

$$
\begin{aligned}
& \Psi_{0}=C_{1313} \leftrightarrow \Psi_{0000}, \quad \Psi_{1}=C_{1213} \leftrightarrow \Psi_{0001}, \quad \Psi_{2}=C_{1342} \leftrightarrow \Psi_{0011}, \\
& \Psi_{3}=C_{1242} \leftrightarrow \Psi_{0111}, \quad \Psi_{4}=C_{2424} \leftrightarrow \Psi_{1111} .
\end{aligned}
$$


Their transformations law under Lorentz rotations can be either worked out directly by using $C_{a_{1} a_{2} a_{3} a_{4}}^{\prime}=\Lambda_{a_{1}}{ }^{b_{1}} \ldots \Lambda_{a_{4}}{ }^{b_{4}} C_{b_{1} b_{2} b_{3} b_{4}}$ and the symmetries of the Weyl tensor, as done in [30] for the individual rotations of type $I, I I, I I I$. A faster way is to use the correspondence with the Weyl spinor: with our choice of Infeld-van der Waerden symbols in (6.4) (cf. (3.1.50) of [39]), $e_{a}=(l, n, m, \bar{m}) \leftrightarrow \epsilon_{A} \bar{\epsilon}_{\dot{A}}=\left(\epsilon_{0} \bar{\epsilon}_{0}, \epsilon_{1} \bar{\epsilon}_{1}, \epsilon_{0} \bar{\epsilon}_{1}, \epsilon_{1} \bar{\epsilon}_{0}\right), e_{a}^{\prime}=\Lambda_{a}^{b} e_{b}$ with $\Lambda_{a}^{b}$ as in (6.9) corresponds to $\epsilon_{A}^{\prime}=g_{A}{ }^{B} \epsilon_{B}$ with

$$
g_{A}{ }^{B}=\left(\begin{array}{cc}
-d & c \\
b & -a
\end{array}\right), \quad g_{B}^{A}=\left(\begin{array}{ll}
a & b \\
c & d
\end{array}\right)
$$

When taking into account the complete symmetry of the Weyl spinor, one gets directly from $\Psi_{A_{1} A_{2} A_{3} A_{4}}^{\prime}=g_{A_{1}}{ }^{B_{1}} \ldots g_{A_{4}}{ }^{B_{4}} \Psi_{B_{1} B_{2} B_{3} B_{4}}$ that

$$
\begin{aligned}
\Psi_{4}^{\prime}= & a^{4} \Psi_{4}-4 a^{3} b \Psi_{3}+6 a^{2} b^{2} \Psi_{2}-4 a b^{3} \Psi_{1}+b^{4} \Psi_{0}, \\
\Psi_{3}^{\prime}= & -a^{3} c \Psi_{4}+\left(a^{3} d+3 a^{2} b c\right) \Psi_{3}-3\left(a b^{2} c+a^{2} b d\right) \Psi_{2} \\
& +\left(b^{3} c+3 a b^{2} d\right) \Psi_{1}-b^{3} d \Psi_{0}, \\
\Psi_{2}^{\prime}= & a^{2} c^{2} \Psi_{4}-2\left(a b c^{2}+a^{2} c d\right) \Psi_{3}+\left(b^{2} c^{2}+4 a b c d+a^{2} d^{2}\right) \Psi_{2} \\
& -2\left(b^{2} c d+a b d^{2}\right) \Psi_{1}+b^{2} d^{2} \Psi_{0}, \\
\Psi_{1}^{\prime}= & -a c^{3} \Psi_{4}+\left(b c^{3}+3 a c^{2} d\right) \Psi_{3}-3\left(b c^{2} d+a c d^{2}\right) \Psi_{2} \\
& +\left(3 b c d^{2}+a d^{3}\right) \Psi_{1}-b d^{3} \Psi_{0}, \\
\Psi_{0}^{\prime}= & c^{4} \Psi_{4}-4 c^{3} d \Psi_{3}+6 c^{2} d^{2} \Psi_{2}-4 c d^{3} \Psi_{1}+d^{4} \Psi_{0} .
\end{aligned}
$$

\subsection{Newman-Unti solution space}

The gauge fixing conditions at null infinity ${ }^{1}$ that are usually assumed correspond to imposing the six real conditions encoded in $\kappa=\epsilon=\pi=0$. This is equivalent to requiring $\Gamma_{a b 1}=0$ and can be achieved by a suitable Lorentz rotation. According to the definition of the Newman-Penrose scalars, it implies that the whole tetrad is parallely transported along $l, D l=0=D n=D m=D \bar{m}$. In particular, this means that $l$ is the generator of affinely parametrized null geodesics. One then requires in addition that $l$ is hypersurface orthonormal and a gradient, which yields 3 more conditions, $\rho=\bar{\rho}$ and $\tau=\bar{\alpha}+\beta$, see, e.g., section 1.9 of [30].

This allows one to choose Bondi coordinates $x^{\mu}=\left(u, r, x^{A}\right), \mu=0, \ldots, 3, A=2,3$, $x^{A}=(\zeta, \bar{\zeta})$ such that the surfaces $u=$ cte are null with normal vector $l, l_{\mu}=\delta_{\mu}^{0}$ and that $r$ is the suitably normalized affine parameter on the null geodesics generated by $l$. The tetrad then takes the form

$$
l=\frac{\partial}{\partial r}, \quad n=\frac{\partial}{\partial u}+U \frac{\partial}{\partial r}+X^{A} \frac{\partial}{\partial x^{A}}, \quad m=\omega \frac{\partial}{\partial r}+\xi^{A} \frac{\partial}{\partial x^{A}},
$$

which implies that

$$
g^{0 \mu}=\delta_{1}^{\mu}, g^{11}=2(U-\omega \bar{\omega}), g^{1 A}=X^{A}-\left(\bar{\omega} \xi^{A}+\omega \bar{\xi}^{A}\right), g^{A B}=-\left(\xi^{A} \bar{\xi}^{B}+\xi^{B} \bar{\xi}^{A}\right)
$$

\footnotetext{
${ }^{1}$ We restrict the discussion to $\mathscr{I}^{+}$.
} 


\begin{tabular}{|c|c|c|c|c|c|c|c|c|c|c|c|c|}
\hline & $\partial$ & $\partial_{u}$ & $\gamma^{0}$ & $\nu^{0}$ & $\mu^{0}$ & $\sigma^{0}$ & $\lambda^{0}$ & $\Psi_{4}^{0}$ & $\Psi_{3}^{0}$ & $\Psi_{2}^{0}$ & $\Psi_{1}^{0}$ & $\Psi_{0}^{0}$ \\
\hline$s$ & 1 & 0 & 0 & -1 & 0 & 2 & -2 & -2 & -1 & 0 & 1 & 2 \\
\hline$w$ & -1 & -1 & -1 & -2 & -2 & -1 & -2 & -3 & -3 & -3 & -3 & -3 \\
\hline
\end{tabular}

Table 1. Spin and conformal weights.

Note furthermore that if $\xi_{A}=g_{A B} \xi^{B}$ with $g_{A B}$ the two dimensional metric inverse to $g^{A B}$, then $\xi^{A} \bar{\xi}_{A}=-1, \xi^{A} \xi_{A}=0=\bar{\xi}^{A} \bar{\xi}_{A}$. The associated cotetrad is given by

$$
\begin{aligned}
& e^{1}=-\left[U+X^{A}\left(\omega \bar{\xi}_{A}+\bar{\omega} \xi_{A}\right)\right] d u+d r+\left(\omega \bar{\xi}_{A}+\bar{\omega} \xi_{A}\right) d x^{A} \\
& e^{2}=d u, \quad e^{3}=X^{A} \bar{\xi}_{A} d u-\bar{\xi}_{A} d x^{A}, \quad e^{4}=X^{A} \xi_{A} d u-\xi_{A} d x^{A} .
\end{aligned}
$$

On a space-like cut of $\mathscr{I}^{+}$, we use coordinates $\zeta, \bar{\zeta}$, and the metric

$$
d \bar{s}^{2}=-\bar{\gamma}_{A B} d x^{A} d x^{B}=-2(P \bar{P})^{-1} d \zeta d \bar{\zeta}
$$

with $P \bar{P}>0$. For the unit sphere, we have $\zeta=\cot \frac{\theta}{2} e^{i \phi}$ in terms of standard spherical coordinates and

$$
P_{S}(\zeta, \bar{\zeta})=\frac{1}{\sqrt{2}}(1+\zeta \bar{\zeta})
$$

The covariant derivative on the 2 surface is then encoded in the operator

$$
\partial \eta^{s}=P \bar{P}^{-s} \bar{\partial}\left(\bar{P}^{s} \eta^{s}\right), \quad \bar{\partial} \eta^{s}=\bar{P} P^{s} \partial\left(P^{-s} \eta^{s}\right),
$$

where $ð, \bar{\partial}$ raise respectively lower the spin weight by one unit. The weights of the various quantities used here are given in table 1. Complex conjugation transforms the spin weight into its opposite and leaves the conformal weight unchanged. Note that $P$ is of spin weight 1 and "holomorphic", $\bar{\partial} P=0$ and that

$$
[\bar{\varnothing}, \check{\partial}] \eta^{s}=\frac{s}{2} R \eta^{s}
$$

with $R=2 P \bar{P} \partial \bar{\partial} \ln (P \bar{P})=2 \bar{\varnothing} \partial \ln (P \bar{P}), R_{S}=2$. We also have

$$
\left[\partial_{u}, \check{\partial}\right] \eta^{s}=\left(\partial_{u} \ln P \partial+s ð \partial_{u} \ln \bar{P}\right) \eta^{s} .
$$

According to [25-27], once the conditions $\kappa=\epsilon=\pi=0$ are fixed and coordinates $u, r, \zeta, \bar{\zeta}$ such that $l_{\nu}=\delta_{\nu}^{u}, l^{\nu}=\delta_{r}^{\nu}$ are chosen, which implies in particular also that $\rho-\bar{\rho}=0=\tau-\bar{\alpha}-\beta$, the leading part of the asymptotic behaviour given in (6.30) follows from the equations of motion, the condition $\Psi_{0}=\Psi_{0}^{0} r^{-5}+O\left(r^{-6}\right)$ and uniform smoothness, i.e., a standard restriction on the functional space imposing how the fall-off conditions in $r$ behave with respect to differentiation. In addition, the choice of a suitable radial coordinate is used to put to zero the term in $\rho$ of order $r^{-2}$, while by a choice of coordinates $x^{A}$, the leading part $r^{2}$ of the spatial metric is set to be conformally flat, and the constant part of $X^{A}$ to vanish. Finally, the leading order $r^{-1}$ of $\tau$ is set to zero by a suitable null rotation. As will be explicitly seen below, these conditions guarantee that the asymptotic symmetry group is the extended BMS group combined with complex rescalings. 
For the explicit form of asymptotic solution space, we will follow closely [27] (see also $[25,26])$, except that the complex $P$ used here is twice the $P$ used there and the $\delta$ operator is taken to agree with the definition used in [33]. Furthermore, $\zeta=x^{3}+i x^{4}$ and $\nabla=2 \bar{\partial}$. More details can be found for instance in the reviews [29, 31, 33, 39] and also in [40], where a translation to results in the BMS gauge as used in [7] can be found. Note also that, as compared to $[7,9,10,40]$, we have changed the signature of the metric in order to agree with the standard conventions used in the context of the Newman-Penrose formalism and that $x^{4} \rightarrow-x^{4}$.

The asymptotic expansion of on-shell spin coefficients, tetrads and the associated components of the Weyl tensor are given by

$$
\begin{aligned}
& \Psi_{0}=\Psi_{0}^{0} r^{-5}+O\left(r^{-6}\right), \\
& \Psi_{1}=\Psi_{1}^{0} r^{-4}-\bar{\partial} \Psi_{0}^{0} r^{-5}+O\left(r^{-6}\right), \\
& \Psi_{2}=\Psi_{2}^{0} r^{-3}-\bar{\varnothing} \Psi_{1}^{0} r^{-4}+O\left(r^{-5}\right), \\
& \Psi_{3}=\Psi_{3}^{0} r^{-2}-\bar{\partial} \Psi_{2}^{0} r^{-3}+O\left(r^{-4}\right), \\
& \Psi_{4}=\Psi_{4}^{0} r^{-1}-\bar{\partial} \Psi_{3}^{0} r^{-2}+O\left(r^{-3}\right), \\
& \rho=-r^{-1}-\sigma^{0} \bar{\sigma}^{0} r^{-3}+O\left(r^{-5}\right), \\
& \sigma=\sigma^{0} r^{-2}+\left(\bar{\sigma}^{0} \sigma^{0} \sigma^{0}-\frac{1}{2} \Psi_{0}^{0}\right) r^{-4}+O\left(r^{-5}\right), \\
& \alpha=\alpha^{0} r^{-1}+\bar{\sigma}^{0} \bar{\alpha}^{0} r^{-2}+\sigma^{0} \bar{\sigma}^{0} \alpha^{0} r^{-3}+O\left(r^{-4}\right), \\
& \beta=-\bar{\alpha}^{0} r^{-1}-\sigma^{0} \alpha^{0} r^{-2}-\left(\sigma^{0} \bar{\sigma}^{0} \bar{\alpha}^{0}+\frac{1}{2} \Psi_{1}^{0}\right) r^{-3}+O\left(r^{-4}\right), \\
& \tau=-\frac{1}{2} \Psi_{1}^{0} r^{-3}+\frac{1}{3}\left(\frac{1}{2} \sigma^{0} \bar{\Psi}_{1}^{0}+\bar{\partial} \Psi_{0}^{0}\right) r^{-4}+O\left(r^{-5}\right), \\
& \lambda=\lambda^{0} r^{-1}-\mu^{0} \bar{\sigma}^{0} r^{-2}+\left(\sigma^{0} \bar{\sigma}^{0} \lambda^{0}+\frac{1}{2} \bar{\sigma}^{0} \Psi_{2}^{0}\right) r^{-3}+O\left(r^{-4}\right), \\
& \mu=\mu^{0} r^{-1}-\left(\sigma^{0} \lambda^{0}+\Psi_{2}^{0}\right) r^{-2}+\left(\sigma^{0} \bar{\sigma}^{0} \mu^{0}+\frac{1}{2} \bar{\delta} \Psi_{1}^{0}\right) r^{-3}+O\left(r^{-4}\right), \\
& \gamma=\gamma^{0}-\frac{1}{2} \Psi_{2}^{0} r^{-2}+\frac{1}{6}\left(2 \bar{\varnothing} \Psi_{1}^{0}+\alpha^{0} \Psi_{1}^{0}-\bar{\alpha}^{0} \bar{\Psi}_{1}^{0}\right) r^{-3}+O\left(r^{-4}\right), \\
& \nu=\nu^{0}-\Psi_{3}^{0} r^{-1}+\frac{1}{2} \bar{\partial} \Psi_{2}^{0} r^{-2}+O\left(r^{-3}\right), \\
& U=-\left(\gamma^{0}+\bar{\gamma}^{0}\right) r+\mu^{0}-\frac{1}{2}\left(\Psi_{2}^{0}+\bar{\Psi}_{2}^{0}\right) r^{-1}+\frac{1}{6}\left(\bar{\partial} \Psi_{1}^{0}+ð \bar{\Psi}_{1}^{0}\right) r^{-2}+O\left(r^{-3}\right), \\
& X^{\zeta}=\overline{X^{\bar{\zeta}}}=\frac{1}{6} \bar{P} \Psi_{1}^{0} r^{-3}+O\left(r^{-4}\right), \\
& \xi^{\zeta}=\overline{\bar{\xi}}=-\bar{P} \sigma^{0} r^{-2}+O\left(r^{-4}\right), \\
& \xi^{\bar{\zeta}}=\overline{\bar{\xi}^{\zeta}}=P\left(r^{-1}+\sigma^{0} \bar{\sigma}^{0} r^{-3}\right)+O\left(r^{-4}\right), \\
& \omega=\bar{\varnothing} \sigma^{0} r^{-1}-\left(\sigma^{0} \precsim \bar{\sigma}^{0}+\frac{1}{2} \Psi_{1}^{0}\right) r^{-2}+O\left(r^{-3}\right),
\end{aligned}
$$




\begin{tabular}{|c|c|c|c|c|c|c|c|c|c|c|c|c|}
\hline & $\bar{\partial}$ & $(P \bar{P})^{-\frac{1}{2}} \partial_{u}$ & $\widetilde{\gamma}^{0}$ & $\widetilde{\nu}^{0}$ & $\widetilde{\mu}^{0}$ & $\widetilde{\sigma}^{0}$ & $\widetilde{\lambda}^{0}$ & $\widetilde{\Psi}_{4}^{0}$ & $\widetilde{\Psi}_{3}^{0}$ & $\widetilde{\Psi}_{2}^{0}$ & $\widetilde{\Psi}_{1}^{0}$ & $\widetilde{\Psi}_{0}^{0}$ \\
\hline$h$ & 0 & $1 / 2$ & $1 / 2$ & $3 / 2$ & 1 & $-1 / 2$ & 2 & $5 / 2$ & 2 & $3 / 2$ & 1 & $1 / 2$ \\
\hline $\bar{h}$ & 1 & $1 / 2$ & $1 / 2$ & $1 / 2$ & 1 & $3 / 2$ & 0 & $1 / 2$ & 1 & $3 / 2$ & 2 & $5 / 2$ \\
\hline
\end{tabular}

Table 2. Conformal dimensions.

where

$$
\begin{aligned}
& \alpha^{0}=\frac{1}{2} \bar{P} \partial \ln P, \\
& \gamma^{0}=-\frac{1}{2} \partial_{u} \ln \bar{P} \\
& \mu_{0}=-\frac{1}{2} \bar{\partial} \partial \ln (P \bar{P})=-\frac{1}{2} P \bar{P} \bar{\partial} \partial \ln (P \bar{P})=-\frac{R}{4}, \\
& \lambda^{0}=\left(\partial_{u}+3 \gamma^{0}-\bar{\gamma}^{0}\right) \bar{\sigma}^{0}, \\
& \nu^{0}=\bar{\varnothing}\left(\gamma^{0}+\bar{\gamma}^{0}\right), \\
& \Psi_{2}^{0}-\bar{\Psi}_{2}^{0}=\bar{\delta}^{2} \sigma^{0}-\partial^{2} \bar{\sigma}^{0}+\bar{\sigma}^{0} \bar{\lambda}^{0}-\sigma^{0} \lambda^{0}, \\
& \Psi_{3}^{0}=-\check{\partial} \lambda^{0}+\bar{\partial} \mu^{0}, \\
& \Psi_{4}^{0}=\bar{ð} \nu^{0}-\left(\partial_{u}+4 \gamma^{0}\right) \lambda^{0},
\end{aligned}
$$

and

$$
\begin{aligned}
\left(\partial_{u}+\gamma^{0}+5 \bar{\gamma}^{0}\right) \Psi_{0}^{0} & =ð \Psi_{1}^{0}+3 \sigma^{0} \Psi_{2}^{0}, \\
\left(\partial_{u}+2 \gamma^{0}+4 \bar{\gamma}^{0}\right) \Psi_{1}^{0} & =ð \Psi_{2}^{0}+2 \sigma^{0} \Psi_{3}^{0}, \\
\left(\partial_{u}+3 \gamma^{0}+3 \bar{\gamma}^{0}\right) \Psi_{2}^{0} & =ð \Psi_{3}^{0}+\sigma^{0} \Psi_{4}^{0} .
\end{aligned}
$$

In this approach to the characteristic initial value problem, freely specifiable initial data at fixed $u_{0}$ is given by $\Psi_{0}\left(u_{0}, r, \zeta, \bar{\zeta}\right)$ in the bulk (with the assumed asymptotics given above) and by $\left(\Psi_{2}^{0}+\bar{\Psi}_{2}^{0}\right)\left(u_{0}, \zeta, \bar{\zeta}\right), \Psi_{1}^{0}\left(u_{0}, \zeta, \bar{\zeta}\right)$ at $\mathscr{I}^{+}$. The asymptotic part of the shear $\sigma^{0}(u, \zeta, \bar{\zeta})$ is free data at $\mathscr{I}^{+}$for all $u$ and determines, together with the other initial data at $\mathscr{I}^{+}$, the would-be conserved BMS currents.

As in [41] (see also [42]), for a field $\eta^{s, w}$ of spin and conformal weights $(s, w)$, one can associate a field $\widetilde{\eta}^{h, \bar{h}}$ of conformal dimensions $(h, \bar{h})$ through

$$
\eta^{s, w}=\bar{P}^{h} P^{\bar{h}} \widetilde{\eta}^{h, \bar{h}}, \quad(h, \bar{h})=\left(-\frac{1}{2}(s+w), \frac{1}{2}(s-w)\right) .
$$

The conformal dimensions of the various quantities used here are given in table 2 .

When expressed in these quantities, (6.34) and (6.35) become

$$
\begin{array}{rlr}
\widetilde{\gamma}^{0}= & -\frac{1}{2}(P \bar{P})^{-\frac{1}{2}} \partial_{u} \ln \bar{P}, \quad \widetilde{\nu}^{0}=\left(\partial+\frac{1}{2} \partial \ln (P \bar{P})\right)\left(\widetilde{\gamma}^{0}+\overline{\widetilde{\gamma}}^{0}\right), \\
\widetilde{\mu}^{0}= & -\frac{1}{2} \partial \bar{\partial} \ln (P \bar{P}), & \widetilde{\lambda}^{0}=(P \bar{P})^{-\frac{1}{2}} \partial_{u} \overline{\widetilde{\sigma}}^{0}, \\
\widetilde{\Psi}_{2}^{0}-\widetilde{\Psi}_{2}^{0}= & \left(\partial^{2}-\frac{1}{2}\left[\partial^{2} \ln (P \bar{P})+\frac{1}{2}(\partial \ln (P \bar{P}))^{2}\right]\right) \widetilde{\sigma}^{0} \\
& -\left(\bar{\partial}^{2}-\frac{1}{2}\left[\bar{\partial}^{2} \ln (P \bar{P})+\frac{1}{2}(\bar{\partial} \ln (P \bar{P}))^{2}\right]\right) \overline{\widetilde{\sigma}}^{0}+\overline{\widetilde{\lambda}}^{0} \overline{\widetilde{\sigma}}^{0}-\widetilde{\lambda}^{0} \widetilde{\sigma}^{0},
\end{array}
$$




$$
\begin{aligned}
& \widetilde{\Psi}_{3}^{0}=-\bar{\partial} \widetilde{\lambda}^{0}+(\partial+\partial \ln (P \bar{P})) \tilde{\mu}^{0}, \\
& \widetilde{\Psi}_{4}^{0}=\left(\partial+\frac{3}{2} \partial \ln (P \bar{P})\right) \widetilde{\nu}^{0}-(P \bar{P})^{-\frac{1}{2}} \partial_{u} \widetilde{\lambda}^{0},
\end{aligned}
$$

and

$$
\begin{aligned}
& (P \bar{P})^{-\frac{1}{2}} \partial_{u} \widetilde{\Psi}_{0}^{0}=(\bar{\partial}+2 \bar{\partial} \ln (P \bar{P})) \widetilde{\Psi}_{1}^{0}+3 \widetilde{\sigma}^{0} \widetilde{\Psi}_{2}^{0}, \\
& (P \bar{P})^{-\frac{1}{2}} \partial_{u} \widetilde{\Psi}_{1}^{0}=\left(\bar{\partial}+\frac{3}{2} \bar{\partial} \ln (P \bar{P})\right) \widetilde{\Psi}_{2}^{0}+2 \widetilde{\sigma}^{0} \widetilde{\Psi}_{3}^{0}, \\
& (P \bar{P})^{-\frac{1}{2}} \partial_{u} \widetilde{\Psi}_{2}^{0}=(\bar{\partial}+\bar{\partial} \ln (P \bar{P})) \widetilde{\Psi}_{3}^{0}+\widetilde{\sigma}^{0} \widetilde{\Psi}_{4}^{0},
\end{aligned}
$$

Below, during the construction of the solution to the evolution equations (6.34) and (6.35), we will construct improved fields of this type that take due care both of the additional $u$-dependence and of the inhomogeneous parts of the transformation laws.

\subsection{Residual gauge symmetries}

The residual gauge symmetries are the combined Lorentz transformations and coordinate changes that leave on-shell spin coefficients and tetrads invariant. Since these transformations map solutions to solutions, once the conditions that determine asymptotic solution space are preserved, no further restrictions can arise. The change of coordinates is of the form

$$
u=u\left(u^{\prime}, r^{\prime}, x^{\prime A}\right), \quad r=r\left(u^{\prime}, r^{\prime}, x^{\prime A}\right), \quad x^{A}=x^{A}\left(u^{\prime}, r^{\prime}, x^{\prime A}\right),
$$

and the unknowns are $A, \bar{A}, B, \bar{B}, E, \bar{E}, u, r, x^{A}$ as functions of $u^{\prime}, r^{\prime}, x^{\prime}$.

Using the $a=1$ component of the transformation law

$$
e_{a}^{\prime \mu} \frac{\partial x^{\nu}}{\partial x^{\prime \mu}}=\Lambda_{a}^{b} e_{b}^{\nu}
$$

it follows that imposing $l^{\prime \mu}=\delta_{r^{\prime}}^{\mu}$ is equivalent to replacing the 1.h.s. by $\frac{\partial x^{\nu}}{\partial r^{\prime}}$. This gives $\frac{\partial x^{\nu}}{\partial r^{\prime}}=d \bar{d} l^{\nu}+c \bar{c} n^{\nu}-\left(d \bar{c} m^{\nu}+\right.$ c.c. $)$, or more explicitly,

$$
\begin{aligned}
\frac{\partial u}{\partial r^{\prime}} & =B \bar{B} e^{-E_{R}} \\
\frac{\partial x^{A}}{\partial r^{\prime}} & =B \bar{B} e^{-E_{R}} X^{A}+\left[\bar{B}(1+\bar{A} B) e^{i E_{I}} \xi^{A}+\text { c.c. }\right], \\
\frac{\partial r}{\partial r^{\prime}} & =|1+A \bar{B}|^{2} e^{E_{R}}+B \bar{B} e^{-E_{R}} U+\left[\bar{B}(1+\bar{A} B) e^{i E_{I}} \omega+\text { c.c. }\right] .
\end{aligned}
$$

In order to implement the gauge fixing conditions in the new coordinate system, or equivalently $\breve{\omega}_{1}^{\prime}=0$, we rewrite the last of (6.17) as

$$
g^{-1} e_{a}^{\prime}(g)=\Lambda_{a}^{b} \breve{\omega}_{b}-g^{-1} \breve{\omega}_{a}^{\prime} g
$$

and require, for $a=1$,

$$
g^{-1} \frac{\partial g}{\partial r^{\prime}}=\Lambda_{1}^{b} \breve{\omega}_{b}
$$


More explicitly, the conditions on the rotation parameters are

$$
d D^{\prime} a-b D^{\prime} c=\Lambda_{1}^{b}\left(\breve{\omega}_{b}\right)_{11}, d D^{\prime} b-b D^{\prime} d=\Lambda_{1}^{b}\left(\breve{\omega}_{b}\right)_{12}, a D^{\prime} c-c D^{\prime} a=\Lambda_{1}{ }^{b}\left(\breve{\omega}_{b}\right)_{21},
$$

where

$$
\begin{aligned}
& \Lambda_{1}^{b}\left(\breve{\omega}_{b}\right)_{11}=c \bar{c} \gamma-d \bar{c} \beta-c \bar{d} \alpha=B \bar{B} e^{-E_{R}} \gamma+\bar{B}(1+\bar{A} B) e^{i E_{I}} \beta+B(1+A \bar{B}) e^{-i E_{I}} \alpha, \\
& \Lambda_{1}^{b}\left(\breve{\omega}_{b}\right)_{12}=c \bar{c} \nu-d \bar{c} \mu-c \bar{d} \lambda=B \bar{B} e^{-E_{R}} \nu+\bar{B}(1+\bar{A} B) e^{i E_{I}} \mu+B(1+A \bar{B}) e^{-i E_{I}} \lambda, \\
& \Lambda_{1}^{b}\left(\breve{\omega}_{b}\right)_{21}=-c \bar{c} \tau+d \bar{c} \sigma+c \bar{d} \rho=-B \bar{B} e^{-E_{R}} \tau-\bar{B}(1+\bar{A} B) e^{i E_{I}} \sigma-B(1+A \bar{B}) e^{-i E_{I}} \rho .
\end{aligned}
$$

Note that the additional equation involving $\Lambda_{1}^{b}\left(\breve{\omega}_{b}\right)_{22}=-\Lambda_{1}^{b}\left(\breve{\omega}_{b}\right)_{11}$ on the r.h.s. follows from the first equation when using $a d-b c=1$. When suitably combining these equations, one finds

$$
\begin{aligned}
& \frac{\partial B}{\partial r^{\prime}}=-e^{E} \Lambda_{1}^{b}\left(\breve{\omega}_{b}\right)_{21}, \\
& \frac{\partial \bar{A}}{\partial r^{\prime}}=-\bar{A}^{2} e^{E} \Lambda_{1}^{b}\left(\breve{\omega}_{b}\right)_{21}-e^{-E} \Lambda_{1}^{b}\left(\breve{\omega}_{b}\right)_{12}, \\
& \frac{\partial E}{\partial r^{\prime}}=2 \bar{A} e^{E} \Lambda_{1}^{b}\left(\breve{\omega}_{b}\right)_{21}-2 \Lambda_{1}^{b}\left(\breve{\omega}_{b}\right)_{11} .
\end{aligned}
$$

The set of equations (6.41) and (6.44) forms a system of differential equations for the radial dependence of the unknown functions. In order to solve it asymptotically, we assume that the functions have the following asymptotic behavior,

$$
r=O\left(r^{\prime}\right), \quad A, E, u, \phi=O(1), \quad B=O\left(r^{\prime-1}\right) .
$$

We can now trade the unknown $r$ in the last of (6.41) for $\chi=r e^{-E_{R}}$ satisfying

$$
\begin{aligned}
& \frac{\partial \chi}{\partial r^{\prime}}=|1+A \bar{B}|^{2}+B \bar{B} e^{-2 E_{R}} U+\left[\bar{B}(1+\bar{A} B) e^{-\bar{E}} \omega\right. \\
&\left.\left.+\chi \Lambda_{1}^{b}\left(\breve{\omega}_{b}\right)_{11}-\chi \bar{A} e^{E} \Lambda_{1}^{b}\left(\breve{\omega}_{b}\right)_{21}\right)+ \text { c.c. }\right]=1+O\left(r^{\prime-2}\right) .
\end{aligned}
$$

Note that the vanishing of the $O\left(r^{\prime-1}\right)$ terms follows from non trivial cancellations. Except for the equation for $r$, which we have just discussed, the r.h.s. of (6.41) and (6.44) are all $O\left(r^{\prime-2}\right)$. We thus have

$$
\begin{aligned}
& A=A_{0}+O\left(r^{\prime-1}\right), \quad r^{\prime} B=B_{0}+O\left(r^{\prime-1}\right), \quad E=E_{0}+O\left(r^{\prime-1}\right), \\
& u=u_{0}+O\left(r^{\prime-1}\right), \quad x^{A}=x_{0}^{A}+O\left(r^{\prime-1}\right), \\
& r=e^{E_{R 0}} r^{\prime}+r_{1}+O\left(r^{\prime-1}\right) \Longleftrightarrow \chi=r^{\prime}+\chi_{1}+\left(r^{\prime-1}\right),
\end{aligned}
$$

where $A_{0}, B_{0}, E_{0}, u_{0}, x_{0}^{A}, r_{1}, \chi_{1}$ are all functions of $u^{\prime}, x^{\prime}$. These fall-offs allow us to write

$$
\begin{aligned}
g^{-1} \frac{\partial g}{\partial r^{\prime}}=\Lambda_{1} \breve{\omega}_{b} & \Longleftrightarrow g\left(u^{\prime}, r^{\prime}, x^{\prime A}\right)=e^{-\int_{r^{\prime}}^{\infty} d \tilde{r}\left(\Lambda_{1}{ }^{b} g \breve{\omega}_{b}\right)\left(u^{\prime}, \tilde{r}, x^{\prime A}\right)} g_{0}\left(u^{\prime}, x^{A}\right), \\
\frac{\partial x^{\mu}}{\partial r^{\prime}}=\Lambda_{1}^{b} e_{b}{ }^{\nu} & \Longleftrightarrow x^{\mu}\left(u^{\prime}, r^{\prime}, x^{A}\right)=x^{\mu}\left(u^{\prime}, \infty, x^{A}\right)-\int_{r^{\prime}}^{\infty} d \tilde{r}\left(\Lambda_{1}^{b} e_{b}{ }^{\mu}\right)\left(u^{\prime}, \tilde{r}, x^{A}\right),
\end{aligned}
$$


for $\mu=u, A$, and where $g_{0}\left(u^{\prime}, x^{\prime A}\right)=g\left(u^{\prime}, \infty, x^{\prime A}\right), x_{0}^{\mu}=x^{\mu}\left(u^{\prime}, \infty, x^{\prime A}\right)$. Even though it will not be explicitly needed in the sequel, equations (6.41) and (6.44) can be used to work out the next to leading orders,

$$
\begin{aligned}
& u=u_{0}-B_{0} \bar{B}_{0} e^{-E_{R 0}} r^{\prime-1}+O\left(r^{\prime-2}\right), \\
& \zeta=\zeta_{0}-B_{0} e^{-E_{0}} \bar{P} r^{\prime-1}+O\left(r^{\prime-2}\right), \\
& B=B_{0} r^{\prime-1}+O\left(r^{\prime-2}\right) \text {, } \\
& A=A_{0}+\left[e^{-2 E_{R 0}+i E_{I 0}} B_{0} \bar{B}_{0} \bar{\nu}^{0}+e^{-2 E_{R 0}} B_{0} \bar{\mu}^{0}+e^{-2 \bar{E}_{0}} \bar{B}_{0} \bar{\lambda}^{0}+A_{0}^{2} \bar{B}_{0}\right] r^{\prime-1}+O\left(r^{\prime-2}\right) \text {, } \\
& E=E_{0}+2\left[B_{0} \bar{B}_{0} e^{-E_{R 0}} \gamma^{0}+B_{0} e^{-E_{0}} \alpha^{0}-\bar{B}_{0} e^{-\bar{E}_{0}} \bar{\alpha}^{0}-\bar{A}_{0} B_{0}\right] r^{\prime-1}+O\left(r^{\prime-2}\right) .
\end{aligned}
$$

At this stage, the unknowns are fixed up to $A_{0}, B_{0}, E_{0}, R_{1}\left(\chi_{1}\right), u_{0}, x_{0}^{A}$ as functions of $u^{\prime}, x^{\prime A}$.

We now have to require $l_{\mu}^{\prime}=\delta_{\mu}^{u^{\prime}}$. After having imposed $l^{\prime}=\frac{\partial}{\partial r^{\prime}}$, one has in particular that $l_{r^{\prime}}^{\prime}=0$. This follows from $l_{r^{\prime}}^{\prime}=\Lambda_{b}^{2} e^{b}{ }_{\nu} \frac{\partial x^{\nu}}{\partial r^{\prime}}$ on the one hand and from $\frac{\partial x^{\nu}}{\partial r^{\prime}}=\Lambda_{1}{ }^{c} e_{c}{ }^{\nu}$ on the other. For the remaining components of $l_{\mu}^{\prime}$ it is enough to verify that $l_{u^{\prime}}^{\prime}=1+o\left(r^{\prime 0}\right)$, $l_{A}^{\prime}=o\left(r^{\prime}\right)$ since solutions are transformed into solutions under local Lorentz and coordinate transformations. In particular, $d e^{\prime a}+\Gamma_{b}^{\prime a} e^{\prime b}=0$, and for $a=2, d e_{1}^{\prime}+\Gamma_{1 b}^{\prime} e^{\prime b}=0$. Contracting with $e_{1}^{\prime \mu}$ then implies that $\partial_{r^{\prime}} l_{\nu^{\prime}}-\partial_{\nu^{\prime}} l_{\mu^{\prime}}^{\prime} e_{1} \mu^{\mu^{\prime}}+\Gamma_{1 b 1}^{\prime} e^{\prime b} \nu_{\nu^{\prime}}-\Gamma_{11 \nu^{\prime}}^{\prime}=0$. This reduces to $\partial_{r^{\prime}} l_{\nu^{\prime}}^{\prime}=\partial_{\nu^{\prime}} l_{r^{\prime}}^{\prime}$, and thus to $\partial_{r^{\prime}} l_{u^{\prime}}^{\prime}=0=\partial_{r^{\prime}} l_{A}^{\prime}$. Extracting the leading order from

$$
\begin{aligned}
e_{\mu}^{\prime 2}=\left[-c \bar{c}\left[U+X^{A}\left(\omega \bar{\xi}_{A}+\bar{\omega} \xi_{A}\right)\right]\right. & \left.+d \bar{d}+c \bar{d} X^{A} \bar{\xi}_{A}+d \bar{c} X^{A} \xi_{A}\right] \frac{\partial u}{\partial x^{\prime \mu}} \\
& +c \bar{c} \frac{\partial r}{\partial x^{\prime \mu}}+\left[c \bar{c}\left(\omega \bar{\xi}_{A}+\bar{\omega} \xi_{A}\right)-c \overline{d \xi}_{A}-d \bar{c} \xi_{A}\right] \frac{\partial x^{A}}{\partial x^{\prime \mu}},
\end{aligned}
$$

we get

$$
\begin{aligned}
& 1=e^{E_{R 0}} \frac{\partial u_{0}}{\partial u^{\prime}}-B_{0} P^{-1} e^{\bar{E}_{0}} \frac{\partial \bar{\zeta}_{0}}{\partial u^{\prime}}-\bar{B}_{0} \bar{P}^{-1} e^{E_{0}} \frac{\partial \zeta_{0}}{\partial u^{\prime}}, \\
& 0=e^{E_{R 0}} \frac{\partial u_{0}}{\partial \zeta^{\prime}}-B_{0} P^{-1} e^{\bar{E}_{0}} \frac{\partial \bar{\zeta}_{0}}{\partial \zeta^{\prime}}-\bar{B}_{0} \bar{P}^{-1} e^{E_{0}} \frac{\partial \zeta_{0}}{\partial \zeta^{\prime}}
\end{aligned}
$$

together with the complex conjugate of the last equation. When using that the change of coordinates needs to be invertible at infinity, these relations are equivalent to

$$
e^{E_{R 0}}=\frac{\partial u_{0}^{\prime}}{\partial u}, \quad B_{0}=-e^{-\bar{E}_{0}} \partial u_{0}^{\prime},
$$

together with the complex conjugate of the last relation.

We now need the transformation laws of $\tau, \sigma$ and $\rho$, which are obtained from the matrix components 21 of the last equation of (6.17) for $a=2,3,4$. This gives

$$
\begin{aligned}
\tau^{\prime}= & a \bar{a}\left(d^{2} \tau+c^{2} \nu-2 c d \gamma\right)-b \bar{a}\left(d^{2} \sigma+c^{2} \mu-2 c d \beta\right)-a \bar{b}\left(d^{2} \rho+c^{2} \lambda-2 c d \alpha\right) \\
& +d \Delta^{\prime}(c)-c \Delta^{\prime}(d) \\
\sigma^{\prime}= & -c \bar{a}\left(d^{2} \tau+c^{2} \nu-2 c d \gamma\right)+d \bar{a}\left(d^{2} \sigma+c^{2} \mu-2 c d \beta\right)+c \bar{b}\left(d^{2} \rho+c^{2} \lambda-2 c d \alpha\right) \\
& +d \delta^{\prime}(c)-c \delta^{\prime}(d)
\end{aligned}
$$




$$
\begin{aligned}
\rho^{\prime}= & -a \bar{c}\left(d^{2} \tau+c^{2} \nu-2 c d \gamma\right)+b \bar{c}\left(d^{2} \sigma+c^{2} \mu-2 c d \beta\right)+a \bar{d}\left(d^{2} \rho+c^{2} \lambda-2 c d \alpha\right) \\
& +d \bar{\delta}^{\prime}(c)-c \bar{\delta}^{\prime}(d)
\end{aligned}
$$

In order to proceed we need the asymptotic behavior of $\Delta, \delta^{\prime}, \bar{\delta}^{\prime}$. Using $e_{a}^{\prime \mu}=\Lambda_{a}^{b} e_{b} \nu \frac{\partial x^{\prime \mu}}{\partial x^{\nu}}$ for $a=2,3$, we get

$$
\begin{aligned}
n^{\prime \mu} & =b \bar{b} D\left(x^{\prime \mu}\right)+a \bar{a} \Delta\left(x^{\prime \mu}\right)-\left[b \bar{a} \delta\left(x^{\prime \mu}\right)+\text { c.c. }\right], \\
m^{\prime \mu} & =-d \bar{b} D\left(x^{\prime \mu}\right)-c \bar{a} \Delta\left(x^{\prime \mu}\right)+d \bar{a} \delta\left(x^{\prime \mu}\right)+c \bar{b} \delta\left(x^{\prime \mu}\right) .
\end{aligned}
$$

Explicitly, this gives

$$
\begin{aligned}
& n^{\prime u^{\prime}}=e^{-E_{R 0}} \frac{\partial u_{0}^{\prime}}{\partial u}+O\left(r^{\prime-1}\right)=1+O\left(r^{\prime-1}\right), \\
& n^{\prime r^{\prime}}=U^{\prime}=e^{-E_{R 0}} \partial_{u}\left(\frac{1}{2} \ln P \bar{P}-E_{R 0}\right) r^{\prime}+O\left(r^{\prime 0}\right), \\
& n^{\prime A}=X^{\prime A}=e^{-E_{R 0}} \frac{\partial x_{0}^{\prime A}}{\partial u}+O\left(r^{\prime-1}\right), \\
& m^{\prime u^{\prime}}=O\left(r^{\prime-1}\right), \\
& m^{\prime r^{\prime}}=\omega^{\prime}=A_{0}+B_{0} e^{-E_{R 0}} \partial_{u}\left(\frac{1}{2} \ln (P \bar{P})-E_{R 0}\right)-e^{-\bar{E}_{0}} \partial E_{R 0}+O\left(r^{\prime-1}\right), \\
& m^{\prime A}=\xi^{\prime A}=\left(B_{0} e^{-E_{R 0}} \frac{\partial x_{0}^{\prime A}}{\partial u}+e^{-\bar{E}_{0}} \partial x_{0}^{\prime A}\right) r^{\prime-1}+O\left(r^{\prime-2}\right) .
\end{aligned}
$$

On-shell the new tetrads need to have the same form in the new coordinates than they had in the old. This implies in particular

$$
\begin{aligned}
\frac{\partial \zeta_{0}^{\prime}}{\partial u} & =0, \quad P^{\prime}=e^{-\bar{E}_{0}} \partial \bar{\zeta}_{0}^{\prime}, \\
A_{0} & =e^{-2 E_{R 0}+i E_{I 0}} \partial_{u}\left(\frac{1}{2} \ln (P \bar{P})-E_{R 0}\right) \partial u_{0}^{\prime}+e^{-\bar{E}_{0}} \precsim E_{R 0},
\end{aligned}
$$

together with the complex conjugates of these equations. In addition the requirement that the leading part of the metric remains conformally flat implies

$$
\frac{\partial \zeta_{0}^{\prime}}{\partial \bar{\zeta}}=0=\frac{\partial \bar{\zeta}_{0}^{\prime}}{\partial \zeta}
$$

When used in (6.49) this leads to

$$
\frac{\partial u_{0}}{\partial u^{\prime}}=e^{-E_{R 0}}, \quad \frac{\partial u_{0}^{\prime}}{\partial \zeta}=-e^{E_{R 0}} \frac{\partial \zeta_{0}^{\prime}}{\partial \zeta} \frac{\partial u_{0}}{\partial \zeta^{\prime}} .
$$

In order to work out the term on the r.h.s. of $\tau^{\prime}$ in (6.51) of order $O\left(r^{\prime-1}\right)$, one needs in particular $n^{\prime r^{\prime}}$ above. Requiring this term to vanish gives

$$
A_{0}=-\partial_{u^{\prime}} B_{0}+B_{0}\left(i \partial_{u^{\prime}} E_{I 0}+\frac{1}{2} e^{-E_{R 0}} \partial_{u} \ln \frac{P}{\bar{P}}\right)
$$

When using (6.50), this coincides with the second of (6.56). 
Requiring that the tems of order $r^{\prime-2}$ in $\rho^{\prime}$ in equation (6.53) vanish yields

$$
\chi_{1}=B_{0} \bar{B}_{0} e^{-E_{R 0}} \partial_{u} \ln (P \bar{P})+A_{0} \bar{B}_{0}+2 \bar{A}_{0} B_{0}+\overline{\bar{\gamma}}^{\prime} B_{0}-2 B_{0} \overline{\bar{\delta}}^{\prime} E_{R 0} .
$$

Finally, to leading order, the transformation law of $\sigma$ in (6.52) yields

$$
\sigma^{\prime 0}=e^{-E_{R 0}+2 i E_{I 0}} \sigma^{0}-A_{0} B_{0}-\check{\partial}^{\prime} B_{0} .
$$

In summary, we see that all the unknowns $A_{0}, \bar{A}_{0}, B_{0}, \bar{B}_{0}, \chi_{1}\left(R_{1}\right), E_{R 0}$ are determined by the change of coordinates at infinity and by $E_{I 0}$. The Jacobian matrices are given by

$$
\begin{gathered}
\left(\begin{array}{ccc}
\frac{\partial u_{0}}{\partial u^{\prime}}=e^{-E_{R 0}} & \frac{\partial u_{0}}{\partial \zeta^{\prime}} & \frac{\partial u_{0}}{\partial \bar{\zeta}^{\prime}} \\
\frac{\partial \zeta_{0}}{\partial u^{\prime}}=0 & \frac{\partial \zeta_{0}}{\partial \zeta^{\prime}}=e^{-\bar{E}_{0}} \frac{\bar{P}}{\bar{P}^{\prime}} & \frac{\partial \bar{\zeta}_{0}}{\partial \bar{\zeta}^{\prime}}=0 \\
\frac{\partial \bar{\zeta}_{0}}{\partial u^{\prime}}=0 & \frac{\partial \bar{\zeta}_{0}}{\partial \zeta^{\prime}}=0 & \frac{\partial \bar{\zeta}_{0}}{\partial \bar{\zeta}^{\prime}}=e^{-\bar{E}_{0} \frac{P}{P^{\prime}}}
\end{array}\right), \\
\left(\begin{array}{ccc}
\frac{\partial u_{0}^{\prime}}{\partial u}=e^{E_{R 0}} & \frac{\partial u_{0}^{\prime}}{\partial \zeta}=-e^{E_{R 0}} \frac{\partial \zeta_{0}^{\prime}}{\partial \zeta} \frac{\partial u_{0}}{\partial \zeta^{\prime}} \frac{\partial u_{0}^{\prime}}{\partial \bar{\zeta}}=-e^{E_{R 0}} \frac{\partial \bar{\zeta}_{0}^{\prime}}{\partial \bar{\zeta}} \frac{\partial u_{0}}{\partial \bar{\zeta}^{\prime}} \\
\frac{\partial \zeta_{0}^{\prime}}{\partial u}=0 & \frac{\partial \zeta_{0}^{\prime}}{\partial \zeta}=e^{E_{0}} \frac{\bar{P}^{\prime}}{\bar{P}} & \frac{\partial \zeta_{0}^{\prime}}{\partial \bar{\zeta}}=0 \\
\frac{\partial \bar{\zeta}_{0}^{\prime}}{\partial u}=0 & \frac{\partial \bar{\zeta}_{0}^{\prime}}{\partial \zeta}=0 & \frac{\partial \bar{\zeta}_{0}^{\prime}}{\partial \bar{\zeta}}=e^{\bar{E}_{0}} \frac{P^{\prime}}{P}
\end{array}\right)
\end{gathered}
$$

Note that here and in the following, when considered as a function of $(u, \zeta, \bar{\zeta}), E_{0}$ is explicitly given by $E_{0}\left(u_{0}^{\prime}(u, \zeta, \bar{\zeta}), \zeta_{0}^{\prime}(\zeta), \bar{\zeta}_{0}^{\prime}(\bar{\zeta})\right)$. Note also that the right lower corner of $(6.62)$ is equivalent to the transformation law of $P$,

$$
P^{\prime}\left(u^{\prime}, \zeta^{\prime}, \bar{\zeta}^{\prime}\right)=P(u, \zeta, \bar{\zeta}) e^{-\bar{E}_{0}} \frac{\partial \bar{\zeta}_{0}^{\prime}}{\partial \bar{\zeta}}
$$

and that preserving $P \bar{P}>0$ requires $\frac{\partial \zeta_{0}^{\prime}}{\partial \zeta} \frac{\partial \bar{\zeta}_{0}^{\prime}}{\partial \bar{\zeta}}>0$. The transformation law of the metric in (6.25) and of $\partial$ are given by

$$
\begin{aligned}
\left(d \bar{s}^{2}\right)^{\prime} & =e^{2 E_{R 0}}\left(d \bar{s}^{2}\right), \\
\check{\partial}^{\prime} \eta^{s} & =e^{-\bar{E}_{0}}\left(ð-e^{-E_{R 0}} \partial u_{0}^{\prime} \partial_{u}-s\left[ð E_{0}-\left(e^{-E_{R 0}} \partial u_{0}^{\prime}\right) \partial_{u}\left(E_{0}-\ln \bar{P}\right)\right]\right) \eta^{s} .
\end{aligned}
$$

In particular, when putting all results together, the subleading term of the rescaled radial coordinate is given by

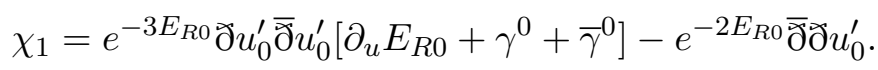

For notational simplicity, we drop in the next sections the subscript 0 on the asymptotic change of coordinates and on the complex Weyl parameter. 


\subsection{Combined extended BMS4 group with complex rescalings}

From the top left corner of the first matrix of (6.62), it follows that $E_{R}$ is determined by $u\left(u^{\prime}, \zeta^{\prime}, \bar{\zeta}^{\prime}\right)$ and, conversely, that the knowledge of such a function allows one to recover the complete change of coordinates, up to an arbitrary function $\hat{u}^{\prime}\left(\zeta^{\prime}, \bar{\zeta}^{\prime}\right)$,

$$
u\left(u^{\prime}, \zeta^{\prime}, \bar{\zeta}^{\prime}\right)=\int_{\hat{u}^{\prime}}^{u^{\prime}} d v^{\prime} e^{-E_{R}}
$$

Note that the point with coordinates $\left(\hat{u}^{\prime}\left(\zeta^{\prime}, \bar{\zeta}^{\prime}\right), \zeta^{\prime}, \bar{\zeta}^{\prime}\right)$ corresponds to $(0, \zeta, \bar{\zeta})$.

After inverting, one can write,

$$
u^{\prime}(u, \zeta, \bar{\zeta})=\int_{\hat{u}}^{u} d v e^{E_{R}}
$$

where $E_{R}=E_{R}\left(u^{\prime}(v, \zeta, \bar{\zeta}), \zeta^{\prime}(\zeta), \bar{\zeta}^{\prime}(\bar{\zeta})\right)$, and now the point with coordinates $(\hat{u}(\zeta, \bar{\zeta}), \zeta, \bar{\zeta})$ is given by $\left(0, \zeta^{\prime}, \bar{\zeta}^{\prime}\right)$ in the new coordinate system.

For a field $P$ transforming as in $(6.63)$, we trade $\hat{u}(\zeta, \bar{\zeta})$ for

$$
\beta(\zeta, \bar{\zeta})=\int_{\hat{u}}^{0} d v(P \bar{P})^{\frac{1}{2}}
$$

which can be inverted since the integrand is positive.

The extended $\mathrm{BMS}_{4}$ group combined with complex rescalings can be parametrized by

$$
\left(\zeta^{\prime}(\zeta), \bar{\zeta}^{\prime}(\bar{\zeta}), \beta(\zeta, \bar{\zeta}), E\left(u^{\prime}, \zeta^{\prime}, \bar{\zeta}^{\prime}\right)\right)
$$

since $\beta(\zeta, \bar{\zeta})$ determines $\hat{u}(\zeta, \bar{\zeta})$ and one then gets $u^{\prime}(u, \zeta, \bar{\zeta})$ from (6.67). Defining

$$
\widetilde{u}(u, \zeta, \bar{\zeta})=\int_{0}^{u} d v(P \bar{P})^{\frac{1}{2}}(v, \zeta, \bar{\zeta}),
$$

its transformation law is simply

$$
\widetilde{u}^{\prime}\left(u^{\prime}, \zeta^{\prime}, \bar{\zeta}^{\prime}\right)=J^{-\frac{1}{2}}[\widetilde{u}(u, \zeta, \bar{\zeta})+\beta(\zeta, \bar{\zeta})], \quad J=\frac{\partial \zeta}{\partial \zeta^{\prime}} \frac{\partial \bar{\zeta}}{\partial \bar{\zeta}^{\prime}}
$$

Together with (6.63), this implies in particular that, if

$$
\beta(\zeta, \bar{\zeta}), \quad E\left(u^{\prime}, \zeta^{\prime}, \bar{\zeta}^{\prime}\right)
$$

and the same quantities with a superscript $s$ and a superscript $c$ are associated to a first, a second successive and the combined transformation respectively, we have

$$
\begin{aligned}
\beta^{c}(\zeta, \bar{\zeta}) & =J^{\frac{1}{2}} \beta^{s}\left(\zeta^{\prime}, \bar{\zeta}^{\prime}\right)+\beta(\zeta, \bar{\zeta}), \\
E^{c}\left(u^{\prime \prime}, \zeta^{\prime \prime}, \bar{\zeta}^{\prime \prime}\right) & =E^{s}\left(u^{\prime \prime}, \zeta^{\prime \prime}, \bar{\zeta}^{\prime \prime}\right)+E\left(u^{\prime}, \zeta^{\prime}, \bar{\zeta}^{\prime}\right) .
\end{aligned}
$$

Alternatively, one can define

$$
\mathcal{U}(u, \zeta, \bar{\zeta})=(P \bar{P})^{-\frac{1}{2}} \widetilde{u}, \quad \alpha(u, \zeta, \bar{\zeta})=(P \bar{P})^{-\frac{1}{2}} \beta,
$$


and parametrize the extended $\mathrm{BMS}_{4}$ combined with complex rescaling through

$$
\left(\zeta^{\prime}(\zeta), \bar{\zeta}^{\prime}(\bar{\zeta}), \alpha(u, \zeta, \bar{\zeta}), E\left(u^{\prime}, \zeta^{\prime}, \bar{\zeta}^{\prime}\right)\right)
$$

Equation (6.71) and the first of equations (6.73) are then replaced by

$$
\begin{aligned}
\mathcal{U}^{\prime}\left(u^{\prime}, \zeta^{\prime}, \bar{\zeta}^{\prime}\right) & =e^{E_{R}\left(u^{\prime}, \zeta^{\prime}, \bar{\zeta}^{\prime}\right)}[\mathcal{U}(u, \zeta, \bar{\zeta})+\alpha(u, \zeta, \bar{\zeta})], \\
\alpha^{c}(u, \zeta, \bar{\zeta}) & =e^{-E_{R}\left(u^{\prime}, \zeta^{\prime}, \bar{\zeta}^{\prime}\right)} \alpha^{s}\left(u^{\prime}, \zeta^{\prime}, \bar{\zeta}^{\prime}\right)+\alpha(u, \zeta, \bar{\zeta}) .
\end{aligned}
$$

A pure complex rescaling is characterized by

$$
\zeta^{\prime}=\zeta, \quad u^{\prime}=\int_{0}^{u} d v e^{E_{R}}, \quad P^{\prime}=P e^{-\bar{E}}
$$

In the case when $P$ does not depend on $u, \widetilde{u}=(P \bar{P})^{\frac{1}{2}} u$ and $\beta=-(P \bar{P})^{\frac{1}{2}} \hat{u}$, whereas $\mathcal{U}=u$ and $\alpha(\zeta, \bar{\zeta})=-\hat{u}$. If furthermore $P^{\prime}$ does not depend on $u^{\prime}$, then neither does $E$ and

$$
u^{\prime}(u, \zeta, \bar{\zeta})=e^{E_{R}\left(\zeta^{\prime}, \bar{\zeta}^{\prime}\right)}(u+\alpha), \quad e^{-E_{R}} \partial u^{\prime}=ð \alpha+ð E_{R}(u+\alpha) .
$$

When the conformal factor is fixed, $P(\zeta, \bar{\zeta})=P_{F}(\zeta, \bar{\zeta})$ and $P^{\prime}\left(\zeta^{\prime}, \bar{\zeta}^{\prime}\right)=P_{F}\left(\zeta^{\prime}, \bar{\zeta}^{\prime}\right)$ for some fixed function $P_{F}$ of its arguments, it follows from (6.63) that complex rescalings are frozen to

$$
e^{E}=\frac{\bar{P}_{F}(\zeta, \bar{\zeta})}{\bar{P}_{F}\left(\zeta^{\prime}, \bar{\zeta}^{\prime}\right)} \frac{\partial \zeta^{\prime}}{\partial \zeta}
$$

In this case, a pure supertranslation is characterized by

$$
\zeta^{\prime}=\zeta, \quad e^{E}=1, \quad u^{\prime}(u, \zeta, \bar{\zeta})=u+\alpha, \quad e^{-E_{R}} \partial u^{\prime}=ð \alpha,
$$

while a pure superrotation is characterized by

$$
\zeta^{\prime}=\zeta^{\prime}(\zeta), e^{E}=\frac{\bar{P}_{F}(\zeta, \bar{\zeta})}{\bar{P}_{F}\left(\zeta^{\prime}, \bar{\zeta}^{\prime}\right)} \frac{\partial \zeta^{\prime}}{\partial \zeta}, u^{\prime}=e^{E_{R}} u, e^{-E_{R}} \partial u^{\prime}=ð E_{R} u .
$$

The standard definition of the $\mathrm{BMS}_{4}$ group is then recovered when (i) standard Lorentz rotations are described through fractional linear transformations (see e.g. [41] for details),

$$
\zeta^{\prime}=\frac{a \zeta+b}{c \zeta+d}, \quad a d-b c=1, \quad a, b, c, d \in \mathbb{C}
$$

(ii) the conformal factor is fixed to be that for the unit sphere, $P_{F}=P_{S}$, in which case

$$
e^{E_{R}^{S}}=\frac{1+\zeta \bar{\zeta}}{(a \zeta+b)(\bar{a} \bar{\zeta}+\bar{b})+(c \zeta+d)(\bar{c} \bar{\zeta}+\bar{d})}, \quad e^{i E_{I}^{S}}=\frac{\bar{c} \bar{\zeta}+\bar{d}}{c \zeta+d}
$$

(iii) supertranslation are expanded in spherical harmonics, $\alpha_{S}=\sum_{l, m} \alpha^{l m} Y_{l m}(\zeta, \bar{\zeta})$ with ordinary translations corresponding to the terms with $l=0,1$ and are explicitly described by

$$
\alpha=\frac{A+B \zeta+\overline{B \zeta}+C \zeta \bar{\zeta}}{1+\zeta \bar{\zeta}}, \quad A, C \in \mathbb{R}, B \in \mathbb{C} .
$$




\subsection{Action on solution space}

Putting the results of the previous subsections together, the transformation law of the data characterizing asymptotic solution space is contained in

$$
\begin{aligned}
& \sigma_{0}^{\prime}=e^{-E_{R}+2 i E_{I}}\left[\sigma_{0}+ð\left(e^{-E_{R}} \partial u^{\prime}\right)-\left(e^{-E_{R}} \partial u^{\prime}\right)\left(\partial_{u}+\bar{\gamma}^{0}-\gamma^{0}\right)\left(e^{-E_{R}} \partial u^{\prime}\right)\right],
\end{aligned}
$$

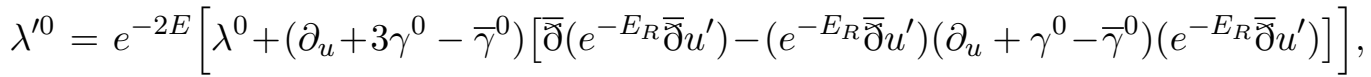

$$
\begin{aligned}
& \Psi_{4}^{\prime 0}=e^{-3 E_{R}-2 i E_{I}} \Psi_{4}^{0}, \\
& \Psi^{\prime 0}{ }_{3}^{0}=e^{-3 E_{R}-i E_{I}}\left[\Psi_{3}^{0}-e^{-E_{R}} \partial u^{\prime} \Psi_{4}^{0}\right], \\
& \Psi_{2}^{\prime 0}=e^{-3 E_{R}}\left[\Psi_{2}^{0}-2 e^{-E_{R}} \partial u^{\prime} \Psi_{3}^{0}+\left(e^{-E_{R}} \partial u^{\prime}\right)^{2} \Psi_{4}^{0}\right], \\
& \Psi_{1}^{\prime 0}=e^{-3 E_{R}+i E_{I}}\left[\Psi_{1}^{0}-3 e^{-E_{R}} \precsim u^{\prime} \Psi_{2}^{0}+3\left(e^{-E_{R}} \partial u^{\prime}\right)^{2} \Psi_{3}^{0}-\left(e^{-E_{R}} \precsim u^{\prime}\right)^{3} \Psi_{4}^{0}\right], \\
& \Psi_{0}^{\prime 0}=e^{-3 E_{R}+2 i E_{I}}\left[\Psi_{0}^{0}-4 e^{-E_{R}} \partial u^{\prime} \Psi_{1}^{0}+6\left(e^{-E_{R}} \partial u^{\prime}\right)^{2} \Psi_{2}^{0}-4\left(e^{-E_{R}} \partial u^{\prime}\right)^{3} \Psi_{3}^{0}+\left(e^{-E_{R}} \partial u^{\prime}\right)^{4} \Psi_{4}^{0}\right] .
\end{aligned}
$$

In particular for instance, if $P, P^{\prime}$ do not depend on $u, u^{\prime}$, the transformation law of the asymptotic shear under a pure supertranslation reduces to

$$
\sigma_{0}^{\prime}=\sigma_{0}+\partial^{2} \alpha, \quad u^{\prime}=u+\alpha, \quad \zeta^{\prime}=\zeta,
$$

while the transformation law of the news under a pure superrotation is

$$
\lambda^{\prime 0}=e^{-2 E}\left[\lambda^{0}+\left(\bar{\partial}^{2} E_{R}-\left(\bar{\partial} E_{R}\right)^{2}\right],\right.
$$

with $u^{\prime}, E$ given in (6.81). If furthermore, we work with respect to the Riemann sphere, $P_{F}=P_{R}=1$, this reduces to

$$
\lambda^{\prime 0}=\left(\frac{\partial \zeta^{\prime}}{\partial \zeta}\right)^{-2}\left[\lambda^{0}+\frac{1}{2}\left\{\zeta^{\prime}, \zeta\right\}\right], \quad u^{\prime}=J^{-\frac{1}{2}} u, \quad \zeta^{\prime}=\zeta^{\prime}(\zeta)
$$

where the Schwarzian derivative is $\{F, x\}=\partial_{x}^{2} \ln \left(\partial_{x} F\right)-\frac{1}{2}\left(\partial_{x} \ln \left(\partial_{x} F\right)\right)^{2}$.

Let us now analyze in more details the evolution equations (6.34). We start with unit scaling factors, $P=\bar{P}=1$, so that in particular the leading part of the metric on a space-like cut of $\mathscr{I}^{+}$is the one on the Riemann sphere, $d \bar{s}^{2}=-2 d \zeta d \bar{\zeta}$. In this case, (6.34) and (6.35) reduce to

$$
\begin{array}{rlrl}
\gamma_{R}^{0} & =0=\nu_{R}^{0}=\mu_{R}^{0}, & \lambda_{R}^{0}=\dot{\bar{\sigma}}_{R}^{0}, \\
\Psi_{2 R}^{0}-\bar{\Psi}_{2 R}^{0} & =\partial^{2} \sigma_{R}^{0}-\bar{\partial}^{2} \bar{\sigma}_{R}^{0}+\dot{\sigma}_{R}^{0} \bar{\sigma}_{R}^{0}-\dot{\bar{\sigma}}_{R}^{0} \sigma_{R}^{0}, \\
\Psi_{3 R}^{0} & =-\bar{\partial} \dot{\bar{\sigma}}_{R}^{0}, & \Psi_{4 R}^{0}=-\ddot{\bar{\sigma}}_{R}^{0},
\end{array}
$$

and

$$
\begin{aligned}
& \partial_{u} \Psi_{0 R}^{0}=\bar{\partial} \Psi_{1 R}^{0}+3 \sigma_{R}^{0} \Psi_{2 R}^{0}, \\
& \partial_{u} \Psi_{1 R}^{0}=\bar{\partial} \Psi_{2 R}^{0}+2 \sigma_{R}^{0} \Psi_{3 R}^{0}, \\
& \partial_{u} \Psi_{2 R}^{0}=\bar{\partial} \Psi_{3 R}^{0}+\sigma_{R}^{0} \Psi_{4 R}^{0},
\end{aligned}
$$


In a first stage, these equations may be trivially solved in terms of integration functions $\widetilde{\Psi}_{a R I}^{0}=\widetilde{\Psi}_{a R I}^{0}(\zeta, \bar{\zeta})$ for $a=0,1,2$ as follows:

$$
\Psi_{2 R}^{0}=\widetilde{\Psi}_{2 R I}^{0}+\int_{0}^{u} d v\left[\bar{\partial} \Psi_{3 R}^{0}+\sigma_{R}^{0} \Psi_{4 R}^{0}\right]
$$

where

$$
\begin{aligned}
\widetilde{\Psi}_{2 R I}^{0}-\widetilde{\bar{\Psi}}_{2 R I}^{0} & =\left(\partial^{2} \sigma_{R}^{0}-\bar{\partial}^{2} \bar{\sigma}_{R}^{0}+\dot{\sigma}_{R}^{0} \bar{\sigma}_{R}^{0}-\dot{\bar{\sigma}}_{R}^{0} \sigma_{R}^{0}\right)(0), \\
\Psi_{1 R}^{0} & =\widetilde{\Psi}_{1 R I}^{0}+\int_{0}^{u} d v\left[\bar{\partial} \Psi_{2 R}^{0}+2 \sigma_{R}^{0} \Psi_{3 R}^{0}\right]
\end{aligned}
$$

and

$$
\Psi_{0 R}^{0}=\widetilde{\Psi}_{0 R I}^{0}+\int_{0}^{u} d v\left[\bar{\partial} \Psi_{1 R}^{0}+3 \sigma_{R}^{0} \Psi_{2 R}^{0}\right]
$$

and the expressions for $\Psi_{1 R}^{0}$ in terms of $\widetilde{\Psi}_{1 R I}^{0}, \widetilde{\Psi}_{2 R I}^{0}, \sigma_{R}^{0}, \dot{\bar{\sigma}}_{R}^{0}$ and for $\Psi_{0 R}^{0}$ in terms of $\widetilde{\Psi}_{0 R I}^{0}, \widetilde{\Psi}_{1 R I}^{0}, \widetilde{\Psi}_{2 R I}^{0}, \sigma_{R}^{0}, \dot{\bar{\sigma}}_{R}^{0}$ can be worked out recursively.

For later use, we introduce instead the integration functions $\Psi_{a R I}^{0}=\Psi_{a R I}^{0}(\zeta, \bar{\zeta})$ for $a=0,1,2$ defined by

$$
\begin{aligned}
\Psi_{2 R}^{0}= & \Psi_{2 R I}^{0}-\bar{\partial}^{2} \bar{\sigma}_{R}^{0}-\sigma_{R}^{0} \dot{\bar{\sigma}}_{R}^{0}+\int_{0}^{u} d v \dot{\sigma}_{R}^{0} \dot{\bar{\sigma}}_{R}^{0}, \quad \Psi_{2 R I}^{0}=\bar{\Psi}_{2 R I}^{0}, \\
\Psi_{1 R}^{0}= & \Psi_{1 R I}^{0}+u \bar{\partial} \Psi_{2 R I}^{0}-\sigma_{R}^{0} \bar{\partial} \bar{\sigma}_{R}^{0}-\frac{1}{2} \bar{\partial}\left(\sigma_{R}^{0} \bar{\sigma}_{R}^{0}\right) \\
& +\int_{0}^{u} d v\left[\frac{1}{2}\left(\bar{\partial} \dot{\sigma}_{R}^{0} \bar{\sigma}_{R}^{0}-3 \sigma_{R}^{0} \bar{\partial} \dot{\bar{\sigma}}_{R}^{0}+3 \dot{\sigma}_{R}^{0} \bar{\partial} \bar{\sigma}_{R}^{0}-\bar{\partial} \sigma_{R}^{0} \dot{\bar{\sigma}}_{R}^{0}\right)-\bar{\partial}^{3} \bar{\sigma}_{R}^{0}\right] \\
& +\bar{\partial} \int_{0}^{u} d v \int_{0}^{v} d w \dot{\sigma}_{R}^{0} \dot{\bar{\sigma}}_{R}^{0}, \\
\Psi_{0 R}^{0}= & \Psi_{0 R I}^{0}+u \bar{\partial} \Psi_{1 R I}^{0}+\frac{1}{2} u^{2} \bar{\partial}^{2} \Psi_{2 R I}^{0} \\
& +\int_{0}^{u} d v\left[\sigma_{R}^{0}\left(3 \Psi_{2 R I}^{0}-3 \sigma_{R}^{0} \dot{\bar{\sigma}}_{R}^{0}-4 \bar{\partial}^{2} \bar{\sigma}_{R}^{0}\right)-2 \bar{\partial} \sigma_{R}^{0} \bar{\partial} \bar{\sigma}_{R}^{0}-\frac{1}{2} \bar{\partial}^{2} \sigma_{R}^{0} \bar{\sigma}_{R}^{0}-\frac{1}{2} \sigma_{R}^{0} \bar{\partial}^{2} \bar{\sigma}_{R}^{0}\right] \\
& +\int_{0}^{u} d v\left[3 \sigma_{R}^{0} \int_{0}^{v} d w \dot{\sigma}_{R}^{0} \dot{\bar{\sigma}}_{R}^{0}\right. \\
& \left.\quad+\bar{\partial} \int_{0}^{v} d w\left[\frac{1}{2}\left(\bar{\partial} \dot{\sigma}_{R}^{0} \bar{\sigma}_{R}^{0}-3 \sigma_{R}^{0} \bar{\partial} \dot{\bar{\sigma}}_{R}^{0}+3 \dot{\sigma}_{R}^{0} \bar{\partial} \bar{\sigma}_{R}^{0}-\bar{\partial} \sigma_{R}^{0} \dot{\bar{\sigma}}_{R}^{0}\right)-\bar{\partial}^{3} \bar{\sigma}_{R}^{0}\right]\right] \\
& +\bar{\partial}^{2} \int_{0}^{u} d v \int_{0}^{v} d w \int_{0}^{w} d x \dot{\sigma}_{R}^{0} \dot{\bar{\sigma}}_{R}^{0} .
\end{aligned}
$$

We have

$$
\begin{aligned}
& \Psi_{2 R I}^{0}=\widetilde{\Psi}_{2 R I}^{0}+\left[\bar{\partial}^{2} \bar{\sigma}_{R}^{0}+\sigma_{R}^{0} \dot{\bar{\sigma}}_{R}^{0}\right](0), \\
& \Psi_{1 R I}^{0}=\widetilde{\Psi}_{1 R I}^{0}+\left[\sigma_{R}^{0} \bar{\partial} \bar{\sigma}_{R}^{0}+\frac{1}{2} \bar{\partial}\left(\sigma_{R}^{0} \bar{\sigma}_{R}^{0}\right)\right](0), \\
& \Psi_{0 R I}^{0}=\widetilde{\Psi}_{0 R I}^{0} .
\end{aligned}
$$


In these terms, the Bondi mass aspect can be chosen to be given by

$$
(-4 \pi G) M_{R}=\Psi_{2 R}^{0}+\sigma_{R}^{0} \dot{\bar{\sigma}}_{R}^{0}+\bar{\partial}^{2} \bar{\sigma}_{R}^{0}=\Psi_{2 R I}^{0}+\int_{0}^{u} d v \dot{\sigma}_{R}^{0} \dot{\bar{\sigma}}_{R}^{0}
$$

Note that this expression contains the additional term $\bar{\partial}^{2} \bar{\sigma}_{R}^{0}$ as compared to the more convential choice, see e.g., equation (4.18) of [10] with $f=1$ (up to a global minus sign due to the change of signature). More details on this quantity, and more generally on the would-be conserved currents and their transformation laws, will be given elsewhere [43].

In order to generate the general solution to (6.34) and (6.35) for arbitrary scaling factors $P, \bar{P}$ from the one with $P=1=\bar{P}$, we apply a pure complex rescaling, without superrotations nor supertranslations to the solution above, i.e., we take $e^{E}=\bar{P}^{\prime-1}, u^{\prime}=$ $\int_{0}^{u} d v e^{E_{R}}, \zeta^{\prime}=\zeta$. In this particular case,

$$
\gamma^{0}=0, e^{-E_{R}} \partial u^{\prime}=-\bar{\partial}^{\prime} u, \partial=\bar{\partial}^{\prime}-\left(P^{\prime} \bar{P}^{\prime}\right)^{-\frac{1}{2}} \bar{\partial}^{\prime} u \partial_{u^{\prime}}, \partial_{u}=\left(P^{\prime} \bar{P}^{\prime}\right)^{-\frac{1}{2}} \partial_{u^{\prime}}
$$

As a consequence, one finds from the transformation laws that the general solution to (6.34) and (6.35) is given by

$$
\begin{aligned}
& \sigma^{0}=\bar{P}^{-\frac{1}{2}} P^{\frac{3}{2}}\left[\sigma_{R}^{0}(\widetilde{u})-\bar{\partial}^{2} \widetilde{u}\right], \\
& \lambda^{0}=\bar{P}^{2}\left[\dot{\bar{\sigma}}_{R}^{0}(\widetilde{u})-\frac{1}{2}\left(\partial^{2} \ln (P \bar{P})+\frac{1}{2}(\partial \ln (P \bar{P}))^{2}\right)\right], \\
& \Psi_{4}^{0}=\bar{P}^{\frac{5}{2}} P^{\frac{1}{2}}\left[\Psi_{4 R}^{0}(\widetilde{u})\right], \\
& \Psi_{3}^{0}=\bar{P}^{2} P\left[\Psi_{3 R}^{0}(\widetilde{u})+\bar{\partial} \widetilde{u} \Psi_{4 R}^{0}(\widetilde{u})\right], \\
& \Psi_{2}^{0}=\bar{P}^{\frac{3}{2}} P^{\frac{3}{2}}\left[\Psi_{2 R}^{0}(\widetilde{u})+2 \bar{\partial} \widetilde{u} \Psi_{3 R}^{0}(\widetilde{u})+(\bar{\partial} \widetilde{u})^{2} \Psi_{4 R}^{0}(\widetilde{u})\right], \\
& \Psi_{1}^{0}=\bar{P} P^{2}\left[\Psi_{1 R}^{0}(\widetilde{u})+3 \bar{\partial} \widetilde{u} \Psi_{2 R}^{0}(\widetilde{u})+3(\bar{\partial} \widetilde{u})^{2} \Psi_{3 R}^{0}(\widetilde{u})+(\bar{\partial} \widetilde{u})^{3} \Psi_{4 R}^{0}(\widetilde{u})\right], \\
& \Psi_{0}^{0}=\bar{P}^{\frac{1}{2}} P^{\frac{5}{2}}\left[\Psi_{0 R}^{0}(\widetilde{u})+4 \bar{\partial} \widetilde{u} \Psi_{1 R}^{0}(\widetilde{u})+6(\bar{\partial} \widetilde{u})^{2} \Psi_{2 R}^{0}(\widetilde{u})\right.
\end{aligned}
$$

where all functions depend on $u, \zeta, \bar{\zeta}$, except where explicitly indicated that the dependence on $u$ is replaced by a dependence on $\widetilde{u}(u, \zeta, \bar{\zeta})$.

In particular, this means that $\sigma_{R}^{0}(\widetilde{u}), \dot{\bar{\sigma}}_{R}^{0}(\widetilde{u}), \Psi_{a R}^{0}(\widetilde{u}), a=0, \ldots, 4$ and $\Psi_{R i I}^{0}, i=0,1,2$, are invariant under complex rescalings. Indeed, applying a complex rescaling to the non reduced quantities amounts to applying the combined complex rescaling to the reduced ones. In other words, only $P, \bar{P}, \widetilde{u}$ change while $\sigma_{R}^{0}, \dot{\bar{\sigma}}_{R}^{0}, \Psi_{a R}^{0}$ are unchanged as a function of their variables, while $\Psi_{i R I}^{0}$ are completely unchanged. More generally, the transformation law of $\sigma_{R}^{0}(\widetilde{u}), \dot{\bar{\sigma}}_{R}^{0}(\widetilde{u}), \Psi_{a R}^{0}(\widetilde{u}), a=0, \ldots, 4$ under the extended BMS group combined with 
complex rescalings simplifies to

$$
\begin{aligned}
\sigma_{R}^{\prime 0} & =\left(\frac{\partial \zeta}{\partial \zeta^{\prime}}\right)^{-\frac{1}{2}}\left(\frac{\partial \bar{\zeta}}{\partial \bar{\zeta}^{\prime}}\right)^{\frac{3}{2}}\left[\sigma_{R}^{0}+\bar{\partial}^{2} \beta+\frac{1}{2}\left\{\bar{\zeta}^{\prime}, \bar{\zeta}\right\}(\tilde{u}+\beta)\right] \\
\dot{\bar{\sigma}}_{R}^{\prime 0} & =\left(\frac{\partial \zeta}{\partial \zeta^{\prime}}\right)^{2}\left[\dot{\bar{\sigma}}_{R}^{0}+\frac{1}{2}\left\{\zeta^{\prime}, \zeta\right\}\right] \\
\Psi_{4 R}^{\prime 0} & =\left(\frac{\partial \zeta}{\partial \zeta^{\prime}}\right)^{\frac{5}{2}}\left(\frac{\partial \bar{\zeta}}{\partial \bar{\zeta}^{\prime}}\right)^{\frac{1}{2}} \Psi_{4 R}^{0}, \\
\Psi_{3 R}^{\prime 0} & =\left(\frac{\partial \zeta}{\partial \zeta^{\prime}}\right)^{2} \frac{\partial \bar{\zeta}}{\partial \bar{\zeta}^{\prime}}\left[\Psi_{3 R}^{0}-Y \Psi_{4 R}^{0}\right], \quad Y=\bar{\partial} \beta+\frac{1}{2} \bar{\partial} \ln \frac{\partial \bar{\zeta}^{\prime}}{\partial \bar{\zeta}}(\tilde{u}+\beta), \\
\Psi_{2 R}^{\prime 0} & =\left(\frac{\partial \zeta}{\partial \zeta^{\prime}}\right)^{\frac{3}{2}}\left(\frac{\partial \bar{\zeta}}{\partial \bar{\zeta}^{\prime}}\right)^{\frac{3}{2}}\left[\Psi_{2 R}^{0}-2 Y \Psi_{3 R}^{0}+Y^{2} \Psi_{4 R}^{0}\right], \\
\Psi_{1 R}^{\prime 0} & =\frac{\partial \zeta}{\partial \zeta^{\prime}}\left(\frac{\partial \bar{\zeta}}{\partial \bar{\zeta}^{\prime}}\right)^{2}\left[\Psi_{1 R}^{0}-3 Y \Psi_{2 R}^{0}+3 Y^{2} \Psi_{3 R}^{0}-Y^{3} \Psi_{4 R}^{0}\right] \\
\Psi_{0 R}^{\prime 0} & =\left(\frac{\partial \zeta}{\partial \zeta^{\prime}}\right)^{\frac{1}{2}}\left(\frac{\partial \bar{\zeta}}{\partial \bar{\zeta}^{\prime}}\right)^{\frac{5}{2}}\left[\Psi_{0 R}^{0}-4 Y \Psi_{1 R}^{0}+6 Y^{2} \Psi_{2 R}^{0}-4 Y^{3} \Psi_{3 R}^{0}+Y^{4} \Psi_{4 R}^{0}\right],
\end{aligned}
$$

where the primed quantities depend on $\widetilde{u}^{\prime}=J^{-\frac{1}{2}}(\widetilde{u}+\beta), \zeta^{\prime}, \bar{\zeta}^{\prime}$, while the unprimed ones depend on $\widetilde{u}, \zeta, \bar{\zeta}$. These transformations simplify for the standard BMS group since the Schwarzian derivative vanishes for this case.

For the transformation law of $\widetilde{\Psi}_{i R I}^{0}$, we find

$$
\begin{aligned}
& \widetilde{\Psi}_{2 R I}^{\prime 0}=\left(\frac{\partial \zeta}{\partial \zeta^{\prime}}\right)^{\frac{3}{2}}\left(\frac{\partial \bar{\zeta}}{\partial \bar{\zeta}^{\prime}}\right)^{\frac{3}{2}}\left[\widetilde{\Psi}_{2 R I}^{0}-\int_{-\beta}^{0} d v\left[\bar{\partial} \Psi_{3 R}^{0}+\sigma_{R}^{0} \Psi_{4 R}^{0}\right]\right. \\
& \left.+\left[-2 \bar{\partial} \beta \Psi_{3 R}^{0}+(\bar{\partial} \beta)^{2} \Psi_{4 R}^{0}\right](-\beta)\right] . \\
& \widetilde{\Psi}_{1 R I}^{\prime 0}=\frac{\partial \zeta}{\partial \zeta^{\prime}}\left(\frac{\partial \bar{\zeta}}{\partial \bar{\zeta}^{\prime}}\right)^{2}\left[\widetilde{\Psi}_{1 R I}^{0}-\int_{-\beta}^{0} d v\left[\bar{\partial} \Psi_{2 R}^{0}+2 \sigma_{R}^{0} \Psi_{3 R}^{0}\right]\right. \\
& \left.+\left[-3 \bar{\partial} \beta \Psi_{2 R}^{0}+3(\bar{\partial} \beta)^{2} \Psi_{3 R}^{0}-(\bar{\partial} \beta)^{3} \Psi_{4 R}^{0}\right](-\beta)\right], \\
& \widetilde{\Psi}_{0 R I}^{\prime 0}=\left(\frac{\partial \zeta}{\partial \zeta^{\prime}}\right)^{\frac{1}{2}}\left(\frac{\partial \bar{\zeta}}{\partial \bar{\zeta}^{\prime}}\right)^{\frac{5}{2}}\left[\widetilde{\Psi}_{0 R I}^{0}-\int_{-\beta}^{0} d v\left[\bar{\partial} \Psi_{1 R}^{0}+3 \sigma_{R}^{0} \Psi_{2 R}^{0}\right]\right. \\
& \left.+\left[-4 \bar{\partial} \beta \Psi_{1 R}^{0}+6(\bar{\partial} \beta)^{2} \Psi_{2 R}^{0}-4(\bar{\partial} \beta)^{3} \Psi_{3 R}^{0}+(\bar{\partial} \beta)^{4} \Psi_{4 R}^{0}\right](-\beta)\right] .
\end{aligned}
$$

The transformation laws of $\Psi_{2 R I}^{0}, \Psi_{1 R I}^{0}$ can be obtained from that of $\widetilde{\Psi}_{2 R I}^{0}, \widetilde{\Psi}_{1 R I}^{0}$ by using the first two relations of (6.100) and equation (B.2), respectively (B.3) of appendix B. This gives

$$
\begin{gathered}
\Psi_{2 R I}^{\prime 0}=\left(\frac{\partial \zeta}{\partial \zeta^{\prime}}\right)^{\frac{3}{2}}\left(\frac{\partial \bar{\zeta}}{\partial \bar{\zeta}^{\prime}}\right)^{\frac{3}{2}}\left[\Psi_{2 R I}^{0}+\bar{\partial}^{2} \partial^{2} \beta+\frac{1}{2}\left\{\bar{\zeta}^{\prime}, \bar{\zeta}\right\}\left(\bar{\sigma}_{R}^{0}+\partial^{2} \beta\right)\right. \\
\left.+\frac{1}{2}\left\{\zeta^{\prime}, \zeta\right\}\left(\sigma_{R}^{0}+\bar{\partial}^{2} \beta\right)-\int_{0}^{\tilde{u}} d \widetilde{v} \dot{\sigma}_{R}^{0} \dot{\bar{\sigma}}_{R}^{0}\right](-\beta)
\end{gathered}
$$




$$
\begin{aligned}
\Psi_{1 R I}^{\prime 0}=\frac{\partial \zeta}{\partial \zeta^{\prime}} & \left(\frac{\partial \bar{\zeta}}{\partial \bar{\zeta}^{\prime}}\right)^{2}\left[\Psi_{1 R I}^{0}-\beta \bar{\partial} \Psi_{2 R I}^{0}-3 \bar{\partial} \beta \Psi_{2 R}^{0}+\frac{3}{2} \bar{\partial}^{2} \beta \bar{\partial} \bar{\sigma}_{R}^{0}+\frac{1}{2} \partial^{2} \beta \bar{\partial} \sigma_{R}^{0}\right. \\
& +\frac{3}{2}\left(\sigma_{R}^{0}+\bar{\partial}^{2} \beta\right)\left(\bar{\partial} \partial^{2} \beta-\bar{\partial} \beta \dot{\bar{\sigma}}_{R}^{0}\right)+\frac{1}{2}\left(\bar{\sigma}_{R}^{0}+\partial^{2} \beta\right)\left(\bar{\partial}^{3} \beta-\bar{\partial} \beta \dot{\sigma}_{R}^{0}\right) \\
& -3(\bar{\partial} \beta)^{2} \bar{\partial}_{R}^{0}+(\bar{\partial} \beta)^{3} \ddot{\bar{\sigma}}_{R}^{0}+\int_{0}^{\tilde{u}} d \widetilde{v} \bar{\partial} \int_{0}^{\widetilde{v}} d \widetilde{w} \dot{\sigma}_{R}^{0} \dot{\bar{\sigma}}_{R}^{0} \\
& \left.+\int_{0}^{\tilde{u}} d \widetilde{v}\left[\frac{1}{2}\left(\bar{\partial} \dot{\sigma}_{R}^{0} \bar{\sigma}_{R}^{0}-3 \sigma_{R}^{0} \bar{\partial}_{\overline{\bar{\sigma}}}^{0}+3 \dot{\sigma}_{R}^{0} \bar{\partial} \bar{\sigma}_{R}^{0}-\bar{\partial} \sigma_{R}^{0} \dot{\bar{\sigma}}_{R}^{0}\right)-\bar{\partial}^{3} \bar{\sigma}_{R}^{0}\right]\right](-\beta) .
\end{aligned}
$$

Finally, the transformation law of the Bondi mass aspect as chosen in (6.101) is given by (1.3) after using (B.4). Note that the transformation law of the standard expression for the Bondi mass aspect can easily be obtained by using (B.1).

\section{Discussion}

In this work, we have generalized finite $\mathrm{BMS}_{4}$ transformations to include general holomorphic and antiholomorphic transformations as well as time-dependent complex rescalings. A further interesting generalization would be to abandon the reality conditions and consider the transformations discussed in this work in the context of $\mathcal{H}$-space [44-46].

The approach we have followed here is systematic and straightforward but explicit computations are rather tedious and can presumably be simplified in a more suitable setup. Extracting physical consequences from these transformation laws should be much more rewarding. We conclude with some comments on why this should be the case.

The residual symmetry group we have investigated acts on the general asymptotically flat solution space in the sense of Newman-Unti [27], containing not only the Kerr black hole [47] but also Robinson-Trautman waves [48, 49]. In this context, the analog of the time coordinate $\widetilde{u}$ used here has been introduced previously in [50] in order to express the latter solutions in terms of a Bondi coordinate system where the conformal factor is the one for the unit sphere.

The transformations also naturally act on the would-be conserved BMS currents including Bondi mass and angular momentum aspects, which are built out of the data considered here. In order to cover the most general case, the expressions considered for instance in [10] have first to be generalized to the case of a variable, complex, $u$-dependent factor $P$. This will be done in [43].

The relevance of the transformation formulas to the gravitational memory effect $[51,52]$ as described in [53] (see [54,55] for recent discussions) is obvious. The question of what part of this effect is controlled by BMS transformations boils down to a question about suitable orbits of the BMS group. These problems will be discussed in more details elsewhere, together with other applications involving topology-changing mappings.

\section{Acknowledgments}

This work is supported in part by the Fund for Scientific Research-FNRS (Belgium), by IISN-Belgium, and by "Communauté française de Belgique - Actions de Recherche Con- 
certées". C. Troessaert is Conicyt (Fondecyt postdoctoral grant 3140125) and Laurent Houart postdoctoral fellow. The Centro de Estudios Científicos (CECs) is funded by the Chilean Government through the Centers of Excellence Base Financing Program of Conicyt. The authors thank Pujian Mao for pointing out relevant references on the memory effect. They are grateful to Per Sundell and his collaborators at the Universidad Andrés Bello (Chile) for hospitality during the final stages of this work.

\section{A Newman-Penrose field equations in $\mathbf{3 d}$}

The Einstein equations in three dimensions can be expressed as

$$
\begin{aligned}
& D \sigma-\delta \kappa=(\epsilon+2 \sigma) \sigma-(\tau-\pi+2 \beta) \kappa, \\
& D \tau-\Delta \kappa=2(\tau+\pi) \sigma-2 \kappa \gamma \\
& D \beta-\delta \epsilon=2(\beta+\pi) \sigma-(2 \mu+\gamma) \kappa-(\beta-\pi) \epsilon, \\
& D \gamma-\Delta \epsilon=2(\tau+\pi) \beta-2 \epsilon \gamma+2 \tau \pi-2 \kappa \nu+\frac{1}{L^{2}}, \\
& D \mu-\delta \pi=(2 \sigma-\epsilon) \mu+\pi^{2}-\kappa \nu-\frac{1}{2 L^{2}}, \\
& D \nu-\Delta \pi=2(\pi+\tau) \mu-2 \epsilon \nu \\
& \Delta \mu-\delta \nu=-(2 \mu+\gamma) \mu+(\pi+2 \beta-\tau) \nu, \\
& \Delta \beta-\delta \gamma=(\beta-\tau) \gamma-2(\beta+\tau) \mu+(2 \sigma+\epsilon) \nu, \\
& \Delta \sigma-\delta \tau=(\gamma-2 \mu) \sigma-\tau^{2}+\nu \kappa+\frac{1}{2 L^{2}},
\end{aligned}
$$

while vanishing of torsion can be written as

$$
\begin{aligned}
D n^{\mu}-\Delta l^{\mu} & =-\gamma l^{\mu}-\epsilon n^{\mu}+2(\pi+\tau) m^{\mu}, \\
D m^{\mu}-\delta l^{\mu} & =(\pi-\beta) l^{\mu}-\kappa n^{\mu}+2 \sigma m^{\mu}, \\
\Delta m^{\mu}-\delta n^{\mu} & =\nu l^{\mu}+(\beta-\tau) n^{\mu}-2 \mu m^{\mu} .
\end{aligned}
$$

\section{B Additional transformation laws in $4 d$}

$$
\begin{aligned}
\bar{\partial}^{\prime 2} \bar{\sigma}_{R}^{\prime 0}= & J^{\frac{3}{2}}\left[\left[\bar{\partial}^{2}+\frac{1}{2}\left\{\bar{\zeta}^{\prime}, \bar{\zeta}\right\}\right] \bar{\sigma}_{R}^{0}+\left[\bar{\partial}^{2}+\frac{1}{2}\left\{\bar{\zeta}^{\prime}, \bar{\zeta}\right\}\right] \partial^{2} \beta\right. \\
& \left.-\left[\bar{\partial}^{2} \beta+\frac{1}{2}\left\{\bar{\zeta}^{\prime}, \bar{\zeta}\right\}(\widetilde{u}+\beta)\right] \dot{\bar{\sigma}}_{R}^{0}-2 Y \bar{\partial} \dot{\bar{\sigma}}_{R}^{0}+Y^{2} \ddot{\bar{\sigma}}_{R}^{0}\right], \\
\bar{\partial}^{\prime 2} \bar{\sigma}_{R}^{\prime 0}+\sigma_{R}^{\prime 0} \dot{\bar{\sigma}}_{R}^{\prime 0}= & J^{\frac{3}{2}}\left[\bar{\partial}^{2} \bar{\sigma}_{R}^{0}+\sigma_{R}^{0} \dot{\bar{\sigma}}_{R}^{0}-2 Y \bar{\partial}_{\bar{\sigma}}^{0}+Y^{2} \ddot{\bar{\sigma}}_{R}^{0}+\frac{1}{2}\left\{\bar{\zeta}^{\prime}, \bar{\zeta}\right\}\left(\bar{\sigma}_{R}^{0}+\partial^{2} \beta\right)\right. \\
& \left.+\frac{1}{2}\left\{\zeta^{\prime}, \zeta\right\}\left(\sigma_{R}^{0}+\bar{\partial}^{2} \beta\right)+\partial^{2} \bar{\partial}^{2} \beta+\frac{1}{4}\left\{\bar{\zeta}^{\prime}, \bar{\zeta}\right\}\left\{\zeta^{\prime}, \zeta\right\}(\widetilde{u}+\beta)\right],
\end{aligned}
$$




$$
\begin{aligned}
\sigma_{R}^{\prime 0} \bar{\partial}^{\prime} \bar{\sigma}_{R}^{\prime 0}+\frac{1}{2} \bar{\partial}^{\prime}\left(\sigma_{R}^{\prime 0} \bar{\sigma}_{R}^{\prime 0}\right)= & \frac{\partial \zeta}{\partial \zeta^{\prime}}\left(\frac{\partial \bar{\zeta}}{\partial \bar{\zeta}^{\prime}}\right)^{2}\left[\frac{3}{2}\left[\sigma_{R}^{0}+\bar{\partial}^{2} \beta+\frac{1}{2}\left\{\bar{\zeta}^{\prime}, \bar{\zeta}\right\}(\widetilde{u}+\beta)\right]\right. \\
& {\left[\bar{\partial} \bar{\sigma}_{R}^{0}+\bar{\partial} \partial^{2} \beta-Y_{\dot{\sigma}_{R}^{0}}^{0}-\frac{1}{4} \bar{\partial} \ln \frac{\partial \bar{\zeta}^{\prime}}{\partial \bar{\zeta}}\left\{\zeta^{\prime}, \zeta\right\}(\widetilde{u}+\beta)\right] } \\
& +\frac{1}{2}\left[\bar{\sigma}_{R}^{0}+\partial^{2} \beta+\frac{1}{2}\left\{\zeta^{\prime}, \zeta\right\}(\widetilde{u}+\beta)\right] \\
& {\left.\left[\bar{\partial} \sigma_{R}^{0}+\bar{\partial}^{3} \beta-Y \dot{\sigma}_{R}^{0}+\frac{1}{2}\left(\bar{\partial}-\frac{1}{2} \bar{\partial} \ln \frac{\partial \bar{\zeta}^{\prime}}{\partial \bar{\zeta}}\right)\left\{\bar{\zeta}^{\prime}, \bar{\zeta}\right\}(\widetilde{u}+\beta)\right]\right], } \\
\left(\int_{0}^{\widetilde{u}} d \widetilde{v} \dot{\sigma}_{R}^{0} \dot{\bar{\sigma}}_{R}^{0}\right)^{\prime}= & J^{\frac{3}{2}}\left[\frac{1}{2}\left\{\bar{\zeta}^{\prime}, \bar{\zeta}\right\}\left(\bar{\sigma}_{R}^{0}-\bar{\sigma}_{R}^{0}(-\beta)\right)+\frac{1}{2}\left\{\zeta^{\prime}, \zeta\right\}\left(\sigma_{R}^{0}-\sigma_{R}^{0}(-\beta)\right)\right. \\
& \left.+\frac{1}{4}\left\{\bar{\zeta}^{\prime}, \bar{\zeta}\right\}\left\{\zeta^{\prime}, \zeta\right\}(\widetilde{u}+\beta)+\int_{-\beta}^{\widetilde{u}} d \widetilde{v} \dot{\sigma}_{R}^{0} \dot{\bar{\sigma}}_{R}^{0}\right] .
\end{aligned}
$$

Open Access. This article is distributed under the terms of the Creative Commons Attribution License (CC-BY 4.0), which permits any use, distribution and reproduction in any medium, provided the original author(s) and source are credited.

\section{References}

[1] A.A. Belavin, A.M. Polyakov and A.B. Zamolodchikov, Infinite Conformal Symmetry in Two-Dimensional Quantum Field Theory, Nucl. Phys. B 241 (1984) 333 [InSPIRE].

[2] C. Itzykson and J. Drouffe, Statistical field theory. Volume 2: strong coupling, Monte Carlo methods, conformal field theory, and random systems, Cambridge University Press, Camrbidge U.K. (1989).

[3] H. Bondi, M.G.J. van der Burg and A.W.K. Metzner, Gravitational waves in general relativity. 7. Waves from axisymmetric isolated systems, Proc. Roy. Soc. Lond. A 269 (1962) 21 [INSPIRE].

[4] R.K. Sachs, Gravitational waves in general relativity. 8. Waves in asymptotically flat space-times, Proc. Roy. Soc. Lond. A 270 (1962) 103 [InSPIRE].

[5] R. Sachs, Asymptotic symmetries in gravitational theory, Phys. Rev. 128 (1962) 2851 [INSPIRE].

[6] G. Barnich and C. Troessaert, Symmetries of asymptotically flat 4 dimensional spacetimes at null infinity revisited, Phys. Rev. Lett. 105 (2010) 111103 [arXiv:0909.2617] [INSPIRE].

[7] G. Barnich and C. Troessaert, Aspects of the BMS/CFT correspondence, JHEP 05 (2010) 062 [arXiv: 1001.1541 ] [INSPIRE].

[8] G. Barnich and C. Troessaert, Supertranslations call for superrotations, PoS (CNCFG2010) 010 [arXiv:1102.4632] [INSPIRE].

[9] G. Barnich and C. Troessaert, BMS charge algebra, JHEP 12 (2011) 105 [arXiv:1106. 0213] [INSPIRE].

[10] G. Barnich and C. Troessaert, Comments on holographic current algebras and asymptotically flat four dimensional spacetimes at null infinity, JHEP 11 (2013) 003 [arXiv:1309.0794] [INSPIRE]. 
[11] A. Strominger, On BMS invariance of gravitational scattering, JHEP 07 (2014) 152 [arXiv: 1312.2229] [INSPIRE].

[12] F. Cachazo and A. Strominger, Evidence for a new soft graviton theorem, arXiv:1404.4091 [INSPIRE].

[13] T. He, V. Lysov, P. Mitra and A. Strominger, BMS supertranslations and Weinberg's soft graviton theorem, JHEP 05 (2015) 151 [arXiv:1401.7026] [INSPIRE].

[14] D. Kapec, V. Lysov, S. Pasterski and A. Strominger, Semiclassical Virasoro symmetry of the quantum gravity $\mathcal{S}$-matrix, JHEP 08 (2014) 058 [arXiv:1406.3312] [INSPIRE].

[15] R. Penrose, Conserved quantities and conformal structure in general relativity, in Relativity theory and astrophysics. 1. Relativity and cosmology, J. Ehlers ed., American Mathematical Society, U.S.A. (1967).

[16] J. Foster, Conformal structure of $i+$ and asymptotic symmetry. I. Definitions and local theory, J. Phys. A 11 (1978) 93.

[17] J. Foster, Asymptotic symmetry and the global structure of future null infinity, Int. J. Theor. Phys. 26 (1987) 1107.

[18] R. Penrose, Asymptotic properties of fields and space-times, Phys. Rev. Lett. 10 (1963) 66 [INSPIRE].

[19] R. Penrose, Zero rest mass fields including gravitation: Asymptotic behavior, Proc. Roy. Soc. Lond. A 284 (1965) 159 [INSPIRE].

[20] M. Bañados, Gravitons and gauge fields in Chern-Simons supergravity, Nucl. Phys. Proc. Suppl. 88 (2000) 17 [hep-th/9911150] [INSPIRE].

[21] K. Skenderis and S.N. Solodukhin, Quantum effective action from the AdS/CFT correspondence, Phys. Lett. B 472 (2000) 316 [hep-th/9910023] [INSPIRE].

[22] G. Barnich, A. Gomberoff and H.A. González, Three-dimensional Bondi-Metzner-Sachs invariant two-dimensional field theories as the flat limit of Liouville theory, Phys. Rev. D 87 (2013) 124032 [arXiv:1210.0731] [INSPIRE].

[23] C. Imbimbo, A. Schwimmer, S. Theisen and S. Yankielowicz, Diffeomorphisms and holographic anomalies, Class. Quant. Grav. 17 (2000) 1129 [hep-th/9910267] [INSPIRE].

[24] A. Schwimmer and S. Theisen, Diffeomorphisms, anomalies and the Fefferman-Graham ambiguity, JHEP 08 (2000) 032 [hep-th/0008082] [INSPIRE].

[25] E. Newman and R. Penrose, An approach to gravitational radiation by a method of spin coefficients, J. Math. Phys. 3 (1962) 566.

[26] E. Newman and R. Penrose, Errata: an approach to gravitational radiation by a method of spin coefficients, J. Math. Phys. 4 (1963) 998.

[27] E.T. Newman and T.W.J. Unti, Behavior of asymptotically flat empty spaces, J. Math. Phys. 3 (1962) 891.

[28] A.R. Exton, E.T. Newman and R. Penrose, Conserved quantities in the Einstein-Maxwell theory, J. Math. Phys. 10 (1969) 1566 [InSPIRE].

[29] E.P. Newman and K.P. Tod, Asymptotically flat space-times, in General relativity and gravitation. 100 years after the birth of Albert Einstein, volume 2, A. Held. ed., Plenum Press (1980). 
[30] S. Chandrasekhar, The mathematical theory of black holes, Oxford University Press, Oxford U.K. (1998).

[31] J. Stewart, Advanced general relativity, Cambridge University Press, Cambridge U.K. (1991).

[32] H. Stephani et al., Exact solutions of Einstein's field equations, Cambridge University Press, Camrbdige U.K. (2003).

[33] R. Penrose and W. Rindler, Spinors and space-time. Volume 2: spinor and twistor methods in space-time geometry, Cambridge University Press, Camrbidge U.K. (1986).

[34] R. Milson and L. Wylleman, Three-dimensional spacetimes of maximal order, Class. Quant. Grav. 30 (2013) 095004 [arXiv: 1210.6920] [INSPIRE].

[35] M. Bañados, Notes on black holes and three-dimensional gravity, AIP Conf. Proc. 490 (1999) 198 [hep-th/9903244] [INSPIRE].

[36] J.D. Brown and M. Henneaux, Central charges in the canonical realization of asymptotic symmetries: an example from three-dimensional gravity, Commun. Math. Phys. 104 (1986) 207 [INSPIRE].

[37] G. Barnich and G. Compère, Classical central extension for asymptotic symmetries at null infinity in three spacetime dimensions, Class. Quant. Grav. 24 (2007) F15 [gr-qc/0610130] [INSPIRE].

[38] G. Barnich and B. Oblak, Notes on the BMS group in three dimensions: II. Coadjoint representation, JHEP 03 (2015) 033 [arXiv:1502.00010] [INSPIRE].

[39] R. Penrose and W. Rindler, Spinors and space-time. Volume 1: two-spinor calculus and relativistic fields, Cambridge University Press, Cambridge U.K. (1984).

[40] G. Barnich and P.-H. Lambert, A note on the Newman-Unti group, Adv. Math. Phys. 2012 (2012) 197385 [arXiv:1102.0589] [INSPIRE].

[41] A. Held, E.T. Newman and R. Posadas, The Lorentz group and the sphere, J. Math. Phys. 11 (1970) 3145.

[42] M. Eastwood and P. Tod, Edth-a differential operator on the sphere, Math. Proc. Cambridge Phil. Soc. 92 (1982) 317.

[43] G. Barnich, P. Mao and C. Troessaert, Transformation laws of would-be conserved BMS currents, in preparation (2016).

[44] E.T. Newman, Heaven and its properties, Gen. Rel. Grav. 7 (1976) 107 [InSPIRE].

[45] M. Ko, E. Newman and K. Tod, H-space and null infinity, in Symposium on the asymptotic structure of space-time, P. Esposito and L. Witten eds., Plenum Press., New York U.S.A. (1977).

[46] M. Ko, M. Ludvigsen, E. Newman and K. Tod, The theory of h-space, Phys. Rept. 71 (1981) 51.

[47] R.P. Kerr, Gravitational field of a spinning mass as an example of algebraically special metrics, Phys. Rev. Lett. 11 (1963) 237 [INSPIRE].

[48] I. Robinson and A. Trautman, Spherical gravitational waves, Phys. Rev. Lett. 4 (1960) 431 [INSPIRE].

[49] I. Robinson and A. Trautman, Some spherical gravitational waves in general relativity, Proc. Roy. Soc. Lond. A 265 (1962) 463 [INSPIRE]. 
[50] T.M. Adamo, C.N. Kozameh and E.T. Newman, Null geodesic congruences, asymptotically flat space-times and their physical interpretation, Living Rev. Rel. 12 (2009) 6 [arXiv:0906.2155] [INSPIRE].

[51] Y. B. Zeldovich and A. Polnarev, Radiation of gravitational waves by a cluster of superdense stars, Sov. Astron. 18 (1974) 17.

[52] D. Christodoulou, Nonlinear nature of gravitation and gravitational wave experiments, Phys. Rev. Lett. 67 (1991) 1486 [INSPIRE].

[53] J. Frauendiener, Note on the memory effect, Class. Quant. Grav. 9 (1992) 1639.

[54] A. Strominger and A. Zhiboedov, Gravitational memory, BMS supertranslations and soft theorems, JHEP 01 (2016) 086 [arXiv:1411.5745] [INSPIRE].

[55] S. Pasterski, A. Strominger and A. Zhiboedov, New gravitational memories, arXiv:1502.06120 [INSPIRE]. 